CALT-68-2546

hep-th/0504203

\title{
Open string integrability and AdS/CFT
}

\author{
Tristan McLoughlin and Ian Swanson \\ California Institute of Technology \\ Pasadena, CA 91125, USA
}

\begin{abstract}
We present a set of long-range Bethe ansatz equations for open quantum strings on $A d S_{5} \times$ $S^{5}$ and demonstrate that they diagonalize bosonic $\mathfrak{s u}(2)$ and $\mathfrak{s l}(2)$ sectors of the theory in the near-pp-wave limit. Results are compared with energy spectra obtained by direct quantization of the open string theory, and we find agreement in this limit to all orders in the 't Hooft coupling $\lambda=g_{\mathrm{YM}}^{2} N_{c}$. We also propose long-range Bethe ansätze for $\mathfrak{s u}(2)$ and $\mathfrak{s l}(2)$ sectors of the dual $\mathcal{N}=2$ defect conformal field theory. In accordance with previous investigations, we find exact agreement between string theory and gauge theory at one- and two-loop order in $\lambda$, but a general disagreement at higher loops. It has been conjectured that the sudden mismatch at three-loop order may be due to long-range interaction terms that are lost in the weak coupling expansion of the gauge theory dilatation operator. These terms are thought to include interactions that wrap around gauge-traced operators, or around the closed-string worldsheet. We comment on the role these interactions play in both open and closed sectors of string states and operators near the pp-wave/BMN limit of the AdS/CFT correspondence.
\end{abstract}

October 7, 2005 


\section{Introduction}

In 2002 Berenstein, Maldacena and Nastase demonstrated that the energy spectrum of type IIB string theory on a pp-wave background geometry can be matched via the AdS/CFT correspondence to the anomalous dimensions of a special class of planar, large $R$-charge operators in $\mathcal{N}=4$ super Yang-Mills (SYM) theory [1]3. This observation sparked a number of detailed tests of AdS/CFT which go beyond the supergravity limit of the string theory. Many of these investigations have probed simplifying limits of Maldacena's original proposal equating IIB superstring theory on $A d S_{5} \times S^{5}$ with $\mathcal{N}=4$ SYM theory in four flat spacetime dimensions [4. It has been realized in the course of these studies that both the string and gauge theory sides of this duality harbor integrable structures associated with quantum spin chains. The discovery of such structures is tantalizing, as it indicates progress toward finding exact solutions within certain sectors of the correspondence. A more immediate benefit is that the arsenal of techniques associated with the Bethe ansatz methodology has been useful for simplifying the computation of both string energies and gauge theory operator dimensions. Building on previous investigations (see, e.g., [5-[16]), we study the integrability of open string states and explore the matchup with corresponding operator dimensions in a dual defect conformal field theory. This allows us to speculate on the role of a particular subset of non-perturbative interactions that have been conjectured to exist in the planar (large- $N_{c}$ ) limit of $\mathcal{N}=4$ SYM theory.

In this paper we will focus on a branch of work involving the so-called near-pp-wave limit of the string theory [17-23]. It was shown in [24 26] that the ten-dimensional ppwave geometry is a consistent supergravity background that can be realized as a largeradius Penrose limit of $A d S_{5} \times S^{5}$. Finite-radius corrections to this limit induce interactions on the string worldsheet that lift the highly degenerate energy spectrum on the pp-wave. These near-pp-wave perturbations were computed for the full supersymmetric string theory in [18.19, where the complete energy spectrum of two-impurity string states (characterized as having two mode excitations on the worldsheet) was compared in this limit to a corresponding set of operator dimensions computed in a perturbative regime of the gauge theory. The string and gauge theory were found to exhibit a remarkably intricate agreement at one- and two-loop order in the 't Hooft coupling $\lambda=g_{\mathrm{YM}}^{2} N_{c}$, but suffer from a general mismatch at three-loop order and beyond. This pattern of agreement at low orders in perturbation theory followed by higher-order disagreement has been shown to repeat itself for the larger and more detailed spectrum of three-impurity string states in [20, and for the complete set of $N$-impurity spectra in [22]. The sudden three-loop disagreement has also appeared in related studies of the AdS/CFT correspondence involving the comparison of semiclassical extended string configurations in anti-de Sitter backgrounds with dual gauge theory operators [27-30].

The matching procedure between string and gauge theory in this setting is presented schematically in figure 1. The prevailing explanation for the higher-loop mismatch is that the comparison of string energies with operator dimensions derived in the perturbative $\lambda$ expansion suffers from an order-of-limits problem [31-33]. In the upper left-hand region we begin with the conjectured equivalence of IIB superstring theory on $A d S_{5} \times S^{5}$ and 
$\mathcal{N}=4$ SYM theory. On the string theory side of the duality one first takes a large-radius Penrose limit by boosting string states in $A d S_{5} \times S^{5}$ to large angular momentum $J$ along an equatorial geodesic in the $S^{5}$ subspace. This large- $J$ limit is combined with the 't Hooft large$N_{c}$ limit, which is taken such that the ratio $N_{c} / J^{2}$ remains fixed and finite. For comparison with perturbative gauge theory, the large- $J$ Penrose limit is followed by an expansion in small $\lambda^{\prime}=g_{\mathrm{YM}}^{2} N_{c} / J^{2}$ (the so-called modified 't Hooft coupling), leading to the lower righthand region in figure 1. On the gauge theory side, operator dimensions are first derived perturbatively in the small- $\lambda$ expansion. The string angular momentum maps to the scalar component $R$ of the $S U(4) R$-charge, so comparison with string theory requires a large$R$ expansion, keeping $N_{c} / R^{2}$ fixed. This leads, via the left-hand route in figure 11 to the matchup with string energies in the lower right-hand corner. The outstanding question is whether the large- $J$ (or large- $R$ ) and small- $\lambda$ (or small- $\lambda^{\prime}$ ) limits are commutative, and whether non-commutativity can account for the mismatch at three-loop order in $\lambda$.

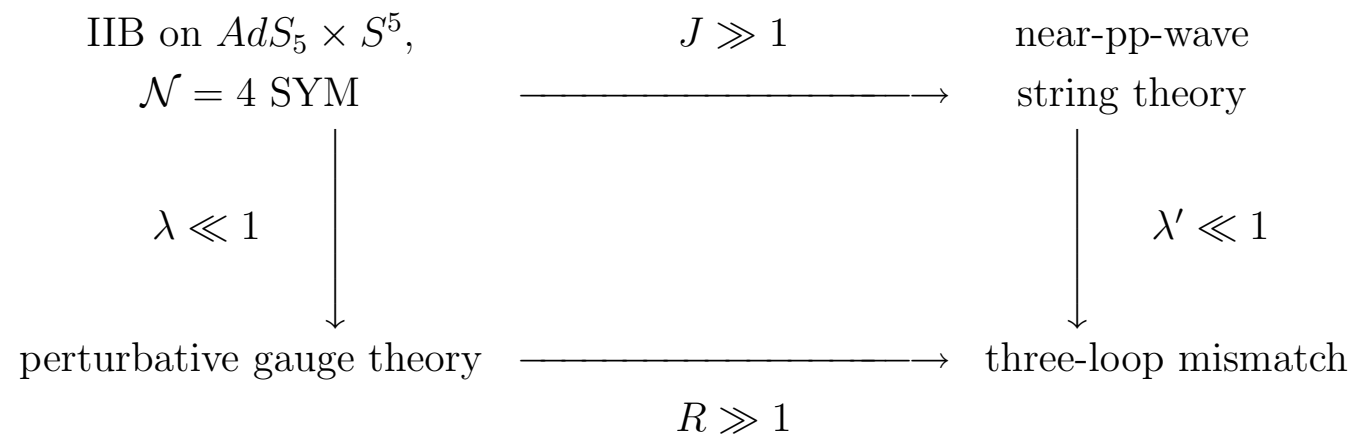

Figure 1: The ordering of limits in the comparison of near-pp-wave string theory with perturbative gauge theory

In the spin chain picture of the gauge theory 34, single-trace operators are identified with cyclic lattice configurations of interacting spins. The excitation of magnon states on the spin lattice corresponds to the insertion of $R$-charge impurities in the trace; this defines a basis on which the dilatation operator, or spin-chain Hamiltonian, can be diagonalized. At one-loop order in $\lambda$ the dilatation operator can be shown to mix only neighboring spins on the lattice (i.e., dilatations of single-trace operators can only mix fields that are adjacent in the trace), and the corresponding statement at $n^{\text {th }}$ order is that the $n$-loop Hamiltonian can mix fields on the lattice that are separated by at most $n$ lattice sites (see figure 2). Within certain sectors of $\mathcal{N}=4 \mathrm{SYM}$ theory, one strategy for extracting operator dimensions at higher loop order in $\lambda$ has been to derive the appropriate $n$-loop spin chain Hamiltonian by enumerating all possible interaction terms of maximum length $n$ and fix the coefficients of these terms using symmetry constraints (or otherwise) handed down from the gauge theory 35-38. One way in which the limits in figure 1 could be non-commutative in this context is that this procedure may neglect the contribution of interactions on the spin lattice that have a range 


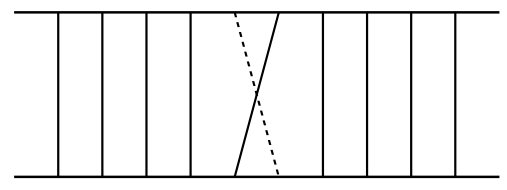

(a) One-loop order

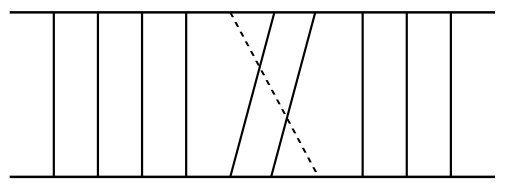

(b) Two-loop order

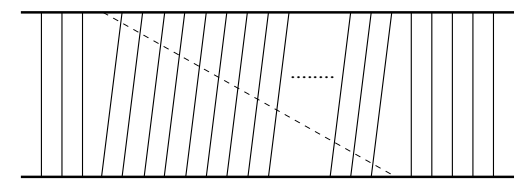

(c) $n$-loop order

Figure 2: Feynman diagrams at (a) one-, (b) two- and (c) $n$-loop order in $\lambda=g_{\mathrm{YM}}^{2} N_{c}$

greater than the total length of the lattice itself 31. The effect of these interactions would be apparent in any calculation that attempts to apply asymptotic Bethe equations (derived for long chains) to spin chains of finite length. This is precisely the situation encountered when comparing finite $R$-charge corrections near the BMN limit to near-pp-wave corrections to the string energy spectrum. The suggestion is that if one were able to eliminate such ultra long-range effects, complete agreement with corresponding string predictions would be obtained.

We attempt to shed light on the specific contribution of wrapping ${ }^{1}$ interactions by studying the matchup of open string states with corresponding gauge theory operators. We formulate a prescription for determining long-range, open-chain Bethe ansätze based on the scattering matrices that appear in the analogous closed-chain equations. On the string side we test our formulas by computing the full, all-loop energy spectrum of open string states in the near-pp-wave limit and comparing with results obtained by direct quantization and diagonalization of the string lightcone Hamiltonian. The string states of interest arise from the IIB theory on $A d S_{5} \times S^{5}$ with a $D 5$ brane wrapping an $A d S_{4} \times S^{2}$ subspace [8, 39]. The spacetime dimensions occupied by both the $D 3$ and $D 5$ branes are indicated in table 1 . Alternatively, one may study an $A d S_{5} \times S^{5} / Z_{2}$ space, which is the near-horizon geometry of

\begin{tabular}{|l|cccccccccc|}
\hline & 0 & 1 & 2 & 3 & 4 & 5 & 6 & 7 & 8 & 9 \\
\hline$D 3$ & $\times$ & $\times$ & $\times$ & & & & & & & $\times$ \\
$D 5$ & $\times$ & $\times$ & $\times$ & $\times$ & $\times$ & $\times$ & & & & \\
\hline
\end{tabular}

Table 1: Extended dimensions of $D 3$ and D5 branes in the ten-dimensional background a large number of D3-branes at an orientifold 7-plane in type $\mathrm{I}^{\prime}$ theory [5]. (The CFT dual is an $S p(N)$ gauge theory.) For the purposes of this paper, however, we find it sufficient to focus on the D3-D5 system, whose dual description is an $\mathcal{N}=2$ defect conformal field theory. We apply our Bethe ansatz on the CFT side of the duality by using the closed (or

\footnotetext{
${ }^{1}$ To be certain, we use the term 'wrapping' here to specify interactions which literally wrap around the lattice.
} 
$\left.\operatorname{protected}^{2}\right) \mathfrak{s u}(2)$ sector of $\mathcal{N}=4$ SYM theory to derive an open-chain, long-range Bethe ansatz for an $\mathfrak{s u}(2)$ sector of operators in the $\mathcal{N}=2$ defect theory.

Let us note here that the mapping of gauge theory physics to the dynamics of integrable open spin chains has been studied in various different settings in the literature. The authors of [6], for example, studied the $S p(N)$ superconformal theory containing one hypermultiplet in the antisymmetric representation and four in the fundamental. This theory had previously been studied in the BMN limit in [5], and its mixing matrix was shown in [6] to map to the Hamiltonian of an integrable open spin chain. Similarly, DeWolfe and Mann [7] considered a general defect conformal field theory [39] (derived as a perturbation to $\mathcal{N}=4 \mathrm{SYM}$ theory) and demonstrated that integrable structures again emerge within certain sectors. This system, which we focus on in this paper, was also examined in [8], where comparisons were carried out at one-loop order between the full pp-wave limit of the string theory and the full BMN limit of the $\mathcal{N}=2$ gauge theory (see also reference [39]). We also note that analyses of semiclassical open string states and corresponding gauge theory operator dimensions were presented in [9]11. The reader is referred to [12, 16, 40] for further related investigations.

In section 2 we establish notation, review the derivation of the bosonic interacting string Hamiltonian in the near-pp-wave limit and compute energy spectra of open string states. This is carried out for a protected $\mathfrak{s u}(2)$ sector of bosonic symmetric-traceless open strings in the $S^{5}$ subspace with Dirichlet boundary conditions, and for an analogous $\mathfrak{s l}(2)$ sector in the $A d S_{5}$ subspace with Neumann boundary conditions. For pedagogical reasons, we treat separately the distinct cases of states with completely unequal mode excitations and those with mode numbers that are allowed to overlap (although we carry this out only for the $\mathfrak{s u}(2)$ sector). In section 3 we formulate a long-range quantum Bethe ansatz for open strings based on long-range scattering matrices developed for corresponding sectors of closed string states in the pure $A d S_{5} \times S^{5}$ background. We solve our resulting Bethe equations in the near-pp-wave limit for general open string states in both the Dirichlet $\mathfrak{s u}(2)$ and Neumann $\mathfrak{s l}(2)$ sectors, and we compare our results with corresponding energy spectra computed directly from the string theory in section 2. In section 4 we apply our long-range Bethe ansatz to the $\mathfrak{s u}(2)$ and $\mathfrak{s l}(2)$ sectors of the dual $\mathcal{N}=2$ defect conformal field theory. The operators of interest are not gauge-traced, and the open-chain Bethe ansätze should be free of any interference from wrapping interactions. Upon solving the Bethe equations in the near-BMN limit, we obtain agreement between operator dimensions in these sectors and the corresponding $\mathfrak{s u}(2)$ and $\mathfrak{s l}(2)$ string energy predictions at one and two-loop order in $\lambda$. Once again, however, this agreement breaks down for both sectors at three-loop order and beyond. We conclude in the final section with a summary and discussion of these results.

\footnotetext{
${ }^{2}$ To avoid confusion with open and closed strings, we will refer to the closed, non-mixing sectors of the string and gauge theory as protected sectors.
} 


\section{Open string energy spectra near the pp-wave limit}

The metric of $A d S_{5} \times S^{5}$ can be written in global coordinates as

$$
d s^{2}=\widehat{R}^{2}\left(-\cosh ^{2} \rho d t^{2}+d \rho^{2}+\sinh ^{2} \rho d \Omega_{3}^{2}+\cos ^{2} \theta d \phi^{2}+d \theta^{2}+\sin ^{2} \theta d \widetilde{\Omega}_{3}^{2}\right),
$$

where the common scale radius of both the $A d S_{5}$ and $S^{5}$ subspaces is denoted by $\widehat{R}, t$ is the global time direction, and $d \Omega_{3}^{2}$ and $d \widetilde{\Omega}_{3}^{2}$ indicate separate three-spheres. As in previous studies, we invoke the following reparameterizations

$$
\cosh \rho=\frac{1+z^{2} / 4}{1-z^{2} / 4}, \quad \cos \theta=\frac{1-y^{2} / 4}{1+y^{2} / 4},
$$

and work with the metric

$$
d s^{2}=\widehat{R}^{2}\left[-\left(\frac{1+\frac{1}{4} z^{2}}{1-\frac{1}{4} z^{2}}\right)^{2} d t^{2}+\left(\frac{1-\frac{1}{4} y^{2}}{1+\frac{1}{4} y^{2}}\right)^{2} d \phi^{2}+\frac{d z_{k} d z_{k}}{\left(1-\frac{1}{4} z^{2}\right)^{2}}+\frac{d y_{k^{\prime}} d y_{k^{\prime}}}{\left(1+\frac{1}{4} y^{2}\right)^{2}}\right]
$$

This version of the spacetime metric is useful when working with fermions (see [19] for details), though we will restrict ourselves to the bosonic sector of the string theory in the present study. The Penrose limit is reached by boosting string states to lightlike momentum $J$ along an equatorial geodesic in the $S^{5}$ subspace. Under the rescaling prescriptions

$$
t \rightarrow x^{+}, \quad \phi \rightarrow x^{+}+\frac{x^{-}}{\widehat{R}^{2}}, \quad z_{k} \rightarrow \frac{z_{k}}{\widehat{R}}, \quad y_{k^{\prime}} \rightarrow \frac{y_{k^{\prime}}}{\widehat{R}}
$$

this limit is reached by taking $\widehat{R} \rightarrow \infty$. The $S^{5}$ angular momentum $J$ is thereby related to the scale radius $\widehat{R}$ by

$$
p_{-} \widehat{R}^{2}=J
$$

and the lightcone momenta are given by

$$
-p_{+}=\Delta-J, \quad-p_{-}=i \partial_{x^{-}}=\frac{i}{\widehat{R}^{2}} \partial_{\phi}=-\frac{J}{\widehat{R}^{2}} .
$$

The transverse Cartesian coordinates $z_{k}$ and $y_{k^{\prime}}$ span an $S O(4) \times S O(4)$ subspace, with $z_{k}$ lying in $A d S_{5}(k \in 1, \ldots, 4)$ and $y_{k^{\prime}}$ in the $S^{5}$ subspace $\left(k^{\prime} \in 5, \ldots, 8\right)$. In the Penrose limit $p_{-}$is held fixed as $J$ and $\widehat{R}$ become infinite. We also work in the planar limit, where the number of colors (or number of $D 3$ branes) $N_{c}$ becomes large, but the quantity $N_{c} / J^{2}$ is held fixed. The lightcone momentum $p_{-}$is equated via the AdS/CFT dictionary with

$$
p_{-}=\frac{1}{\sqrt{\lambda^{\prime}}}=\frac{J}{\sqrt{g_{\mathrm{YM}}^{2} N_{c}}},
$$

where, as noted in the introduction, $\lambda^{\prime}$ is known as the modified 't Hooft coupling, and $J$ is equated on the CFT side with the scalar $R$-charge $R$. 
For reasons described in [18, 19, the lightcone coordinates in eqn. (2.4) admit many simplifications, the most important of which is the elimination of normal-ordering contributions to the lightcone Hamiltonian (at least to the order of interest). In terms of these coordinates, we obtain the following large- $\widehat{R}$ expansion of the metric:

$$
\begin{aligned}
d s^{2} & =2 d x^{+} d x^{-}+d z^{2}+d y^{2}-\left(z^{2}+y^{2}\right)\left(d x^{+}\right)^{2} \\
& +\frac{1}{\widehat{R}^{2}}\left[-2 y^{2} d x^{-} d x^{+}+\frac{1}{2}\left(y^{4}-z^{4}\right)\left(d x^{+}\right)^{2}+\left(d x^{-}\right)^{2}+\frac{1}{2} z^{2} d z^{2}-\frac{1}{2} y^{2} d y^{2}\right]+O\left(1 / \widehat{R}^{2}\right),
\end{aligned}
$$

where the coordinates $x^{A}$ span an $S O(8)$ subspace, with $A \in 1, \ldots, 8$. The pp-wave metric emerges at leading order, and the string theory on that background is free [41,42. The background curvature correction appearing at $O\left(1 / \widehat{R}^{2}\right)$ induces a set of interaction corrections to the free string spectrum in the Penrose limit. As noted in the introduction, these corrections lift the degeneracy of the free theory on the pp-wave, allowing for much more detailed comparisons of string energy spectra with gauge theory anomalous dimensions.

In the Penrose limit, the lightcone Green-Schwarz action for open superstrings takes the form

$$
S_{\mathrm{pp}}=\frac{1}{2 \pi} \int d \tau \int_{0}^{2 \pi} d \sigma\left(\mathcal{L}_{B}+\mathcal{L}_{F}\right)
$$

where

$$
\begin{aligned}
& \mathcal{L}_{B}=\frac{1}{2}\left[\left(\dot{x}^{A}\right)^{2}-\left(x^{\prime A}\right)^{2}-\left(x^{A}\right)^{2}\right], \\
& \mathcal{L}_{F}=i \psi^{\dagger} \dot{\psi}+\psi^{\dagger} \Pi \psi+\frac{i}{2}\left(\psi \psi^{\prime}+\psi^{\dagger} \psi^{\prime \dagger}\right) .
\end{aligned}
$$

(We have set $\alpha^{\prime}=1$.) The fields $\psi_{\alpha}$ are eight-component complex spinors formed from two Majorana-Weyl $S O(9,1)$ spinors of equal chirality, and $\Pi$ is defined in terms of eightdimensional $S O(8)$ Dirac gamma matrices by $\Pi \equiv \gamma^{1} \bar{\gamma}^{2} \gamma^{3} \bar{\gamma}^{4}$ (see [19] for further details). The shorthand notation $\dot{x}^{A}$ and $x^{\prime A}$ denotes the worldsheet derivatives $\partial_{\tau} x^{A}$ and $\partial_{\sigma} x^{A}$, respectively. The pp-wave lightcone Hamiltonian is easily derived from eqns. (2.10) and (2.11):

$$
H_{\mathrm{pp}}=\frac{p_{-}}{2}\left(x^{A}\right)^{2}+\frac{1}{2 p_{-}}\left[\left(p_{A}\right)^{2}+\left(x^{A}\right)^{2}\right]+i \rho \Pi \psi+\frac{i}{2} \psi \psi^{\prime}-\frac{i}{2 p_{-}^{2}} \rho \rho^{\prime},
$$

where the fields $p_{A}$ and $\rho_{\alpha}$ are conjugate to $x^{A}$ and $\psi_{\alpha}$, respectively.

Including the first finite-radius curvature correction to the spacetime metric in eqn. (2.8) gives rise to an interacting Hamiltonian appearing at $O\left(1 / \widehat{R}^{2}\right)$ in the large-radius expansion. This Hamiltonian was computed and analyzed extensively in [18 20, 22]. The bosonic sector, 
labeled by $H_{\mathrm{BB}}$, is quartic in fields and mixes purely bosonic string states:

$$
\begin{aligned}
H_{\mathrm{BB}}= & \frac{1}{\widehat{R}^{2}}\left\{\frac{1}{4 p_{-}}\left[-y^{2}\left(p_{z}^{2}+z^{\prime 2}+2 y^{\prime 2}\right)+z^{2}\left(p_{y}^{2}+y^{\prime 2}+2 z^{\prime 2}\right)\right]+\frac{p_{-}}{8}\left[\left(x^{A}\right)^{2}\right]^{2}\right. \\
& \left.-\frac{1}{8 p_{-}^{3}}\left[\left[\left(p_{A}\right)^{2}\right]^{2}+2\left(p_{A}\right)^{2}\left(x^{\prime A}\right)^{2}+\left[\left(x^{\prime A}\right)^{2}\right]^{2}\right]+\frac{1}{2 p_{-}^{3}}\left(x^{\prime A} p_{A}\right)^{2}\right\} .
\end{aligned}
$$

We have used the shorthand symbols $p_{z}$ and $p_{y}$ to denote bosonic momentum fields in the $A d S_{5}$ and $S^{5}$ subspaces, respectively. Since we will not be dealing with fermions, the string states of interest are formed by acting with bosonic creation operators $a_{n}^{A \dagger}$ on a vacuum state $|J\rangle$ carrying $J$ units of angular momentum in the $S^{5}$ subspace:

$$
|N ; J\rangle \equiv \underbrace{a_{n_{1}}^{A_{1} \dagger} a_{n_{2}}^{A_{2} \dagger} \cdots a_{n_{N}}^{A_{N} \dagger}}_{N}|J\rangle .
$$

Such states are generically referred to as $N$-impurity bosonic string states.

In [18 20] it was demonstrated that there are certain special sectors of the full interacting Hamiltonian that completely decouple from the theory. Among these are two bosonic sectors consisting of pure-boson states restricted to either the $A d S_{5}$ or $S^{5}$ subspaces and projected onto symmetric-traceless irreps of spacetime $S O(4)$, plus one sector comprised of purely fermionic excitations symmetrized in spinor indices (symmetrized spinors survive in this setting because they come with additional mode-number labels). For low impurity number, these sectors are conveniently labeled according to their transformation properties under the residual $S O(4)_{A d S} \times S O(4)_{S^{5}}$ symmetry that survives in the Penrose (or near-Penrose) limit. Employing the $S U(2)^{2} \times S U(2)^{2}$ notation used extensively in [18 20], the two-impurity bosonic sectors transform as $(\mathbf{3}, \mathbf{3} ; \mathbf{1}, \mathbf{1})$ and $(\mathbf{1}, \mathbf{1} ; \mathbf{3}, \mathbf{3})$, while the two-impurity fermionic sectors are labeled by $(\mathbf{3}, \mathbf{1} ; \mathbf{3}, \mathbf{1})$ and $(\mathbf{1}, \mathbf{3} ; \mathbf{1}, \mathbf{3})$. (The three-impurity version of this irrep decomposition is given in [20].) On the gauge theory side the symmetric-traceless $\operatorname{Ad} S_{5}$ $(\mathbf{3}, \mathbf{3} ; \mathbf{1}, \mathbf{1})$ bosons map to a closed sector of the dilatation generator invariant under an $\mathfrak{s l}(2)$ subalgebra of the full superconformal algebra $\mathfrak{p s u}(2,2 \mid 4)$. The protected sector of $(\mathbf{1}, \mathbf{1} ; \mathbf{3}, \mathbf{3})$ bosonic $S^{5}$ string states maps to an $\mathfrak{s u}(2)$ sector, and the $(\mathbf{3}, \mathbf{1} ; \mathbf{3}, \mathbf{1})+(\mathbf{1}, \mathbf{3} ; \mathbf{1}, \mathbf{3})$ fermions correspond to an $\mathfrak{s u}(1 \mid 1)$ sector. We will use these algebraic labels when we refer to these decoupled subsectors in both the string theory and the gauge theory.

Since we are interested in general multi-impurity states within these protected subsectors, we will restrict ourselves in the $\mathfrak{s l}(2)$ sector to open strings with Neumann boundary conditions, and the $\mathfrak{s u}(2)$ sector will be comprised of open strings with Dirichlet boundary conditions. The available bosonic worldsheet fields are indicated in table 2] where, for ex-

ample, $z_{N}^{j}$ indicates fields excited in the $A d S_{5}$ subspace with Neumann boundary conditions, and $y_{D}^{j^{\prime}}$ are $S^{5}$ fluctuations with Dirichlet boundary conditions.

\subsection{Dirichlet $S O(4)_{S^{5}}(\mathfrak{s u}(2))$ sector}

Since the goal is to compute $O(1 / J)$ interaction corrections to the free energy spectrum in the pp-wave limit, we find it convenient to isolate such corrections according to the energy 


\begin{tabular}{|cc|cccc|cccc|}
\hline+ & - & 1 & 2 & 3 & 4 & 5 & 6 & 7 & 8 \\
\hline$x^{+}$ & $x^{-}$ & $z_{N}^{j}$ & $z_{N}^{j}$ & $z_{N}^{j}$ & $z_{D}^{j}$ & $y_{N}^{j^{\prime}}$ & $y_{D}^{j^{\prime}}$ & $y_{D}^{j^{\prime}}$ & $y_{D}^{j^{\prime}}$ \\
\hline
\end{tabular}

Table 2: Neumann and Dirichlet directions on the $S O(4)_{A d S} \times S O(4)_{S^{5}}$ transverse subspace expansion

$$
E\left(\left\{n_{j}\right\}, N, J\right)=\sum_{j=1}^{N} \sqrt{1+n_{j}^{2} \lambda^{\prime} / 4}+\delta E\left(\left\{n_{j}\right\}, N, J\right)+O\left(1 / J^{2}\right) .
$$

The near-pp-wave energy spectra we are interested in are thus collected into the $O(1 / J)$ correction $\delta E\left(\left\{n_{j}\right\}, N, J\right)$, which generically depends on the set of mode numbers $\left\{n_{j}\right\}$ carried by the corresponding (unperturbed) energy eigenstate, the total number of worldsheet mode excitations $N$, and the string $S^{5}$ angular momentum $J$.

We will focus first on a set of $N$-impurity bosonic open string states in the protected $\mathfrak{s u}(2)$ sector. We form symmetric-traceless (in $S O(4)_{S^{5}}$ indices) states by combining excitations in the Dirichlet $\left(y^{6}, y^{7}, y^{8}\right) S^{5}$ directions:

$$
|N ; J\rangle_{D}=a_{n_{1}}^{\left(j_{1}^{\prime} \dagger\right.} a_{n_{2}}^{j_{2}^{\prime} \dagger} \cdots a_{n_{N}}^{\left.j_{N}^{\prime}\right) \dagger}|J\rangle
$$

where the $S^{5}$ labels $j^{\prime}$ indicate the $\left(y^{6}, y^{7}, y^{8}\right)$ directions and contributions to the traced state are understood to be absent.

The Fourier expansion of bosonic fluctuations in the Dirichlet directions is given by [4]

$$
x_{D}^{A}(\tau, \sigma)=\sum_{n=1}^{\infty} \frac{i}{\sqrt{\omega_{n}}} \sin \left(\frac{n \sigma}{2}\right)\left(a_{n}^{A} e^{-i \omega_{n} \tau}-a_{n}^{A \dagger} e^{i \omega_{n} \tau}\right) .
$$

The equations of motion in the pp-wave limit

$$
\ddot{x}^{A}-x^{\prime \prime}+p_{-}^{2} x^{A}=0
$$

are therefore satisfied by the dispersion relation

$$
\omega_{n}=\sqrt{p_{-}^{2}+(n / 2)^{2}}
$$

where the mode index $n$ is integer-valued and the oscillators $a_{n}^{A}$ and $a_{n}^{A \dagger}$ satisfy the usual commutation relations: $\left[a_{n}^{A}, a_{m}^{B \dagger}\right]=\delta_{n, m} \delta^{A, B}$. We are interested in computing diagonal matrix elements of $H_{\mathrm{BB}}$ between physical string states which, in first-order perturbation theory, will involve equal numbers of creation and annihilation operators (i.e., we need not be concerned with sectors of $H_{\mathrm{BB}}$ that mix states with different numbers of impurities). We may therefore restrict to terms appearing in $H_{\mathrm{BB}}$ with two creation and two annihilation operators. 
Following the approach in 22, we simplify the projection onto symmetric-traceless $S^{5}$ string states by forming the following bosonic oscillators:

$$
a_{n} \equiv \frac{1}{\sqrt{2}}\left(a_{n}^{6}+i a_{n}^{7}\right), \quad \bar{a}_{n} \equiv \frac{1}{\sqrt{2}}\left(a_{n}^{6}-i a_{n}^{7}\right)
$$

Generic matrix elements of the form

$$
\left\langle J\left|a_{n_{1}} a_{n_{2}} \cdots a_{n_{N}}\left(H_{\mathrm{BB}}\right) a_{n_{1}}^{\dagger} a_{n_{2}}^{\dagger} \cdots a_{n_{N}}^{\dagger}\right| J\right\rangle
$$

therefore select out excitations in the $\left(y_{D}^{6}, y_{D}^{7}\right)$ plane and make the symmetric-traceless projection manifest. Restricting to the Dirichlet directions, we are also free to project onto the $\left(y_{D}^{6}, y_{D}^{8}\right)$ and $\left(y_{D}^{7}, y_{D}^{8}\right)$ planes. We exclude the $y_{N}^{5}$ direction because we do not want to mix Neumann and Dirichlet boundary conditions (although, in general, such states are certainly allowed), and states in the $S^{5}$ subspace built strictly from $y_{N}^{5}$ excitations would be projected out by the traceless condition.

As described in [22], the oscillator expansion of $H_{\mathrm{BB}}$ admits two generic structures: one characterized by a contraction of $S O(4)$ indices attached to pairs of creation or annihilation operators

$$
a_{n}^{\dagger A} a_{m}^{\dagger A} a_{l}^{B} a_{p}^{B}
$$

and one containing pairs of contracted creation and annihilation operators

$$
a_{n}^{\dagger A} a_{l}^{\dagger B} a_{m}^{A} a_{p}^{B} .
$$

(The indices $\{n, l, m, p\}$ are mode numbers appearing in the mode expansion of $H_{\mathrm{BB}}$.) Expanding in the fields defined in eqn. (2.19), we obtain

$$
\begin{aligned}
& \left.a_{n}^{\dagger A} a_{m}^{\dagger A} a_{l}^{B} a_{p}^{B}\right|_{(6,7)}=\left(a_{n}^{\dagger} \bar{a}_{m}^{\dagger}+\bar{a}_{n}^{\dagger} a_{m}^{\dagger}\right)\left(a_{l} \bar{a}_{p}+\bar{a}_{l} a_{p}\right) \\
& \left.a_{n}^{\dagger A} a_{l}^{\dagger B} a_{m}^{A} a_{p}^{B}\right|_{(6,7)}=\bar{a}_{n}^{\dagger} \bar{a}_{l}^{\dagger} \bar{a}_{m} \bar{a}_{p}+a_{n}^{\dagger} a_{l}^{\dagger} a_{m} a_{p} .
\end{aligned}
$$

Only the second term in eqn. (2.22) will contribute to the matrix elements in (2.20). We may therefore simplify the calculation of the energy spectrum in this sector of the theory by projecting the Hamiltonian onto terms containing only the oscillator structure $a_{n}^{\dagger} a_{l}^{\dagger} a_{m} a_{p}$.

To begin we will compute energy eigenvalues for the simplest eigenstates in which all mode numbers of the symmetric-traceless state

$$
a_{n_{1}}^{\dagger} a_{n_{2}}^{\dagger} \cdots a_{n_{N}}^{\dagger}|J\rangle
$$

are taken to be unequal $\left(n_{1} \neq n_{2} \neq \cdots \neq n_{N}\right)$. Between these states, the interaction Hamiltonian will admit matrix elements whose structure is defined by

$$
\begin{aligned}
& \left\langle J\left|a_{n_{1}} a_{n_{2}} \ldots a_{N_{B}}\left(a_{n}^{\dagger} a_{l}^{\dagger} a_{m} a_{p}\right) a_{n_{1}}^{\dagger} a_{n_{2}}^{\dagger} \ldots a_{N_{B}}^{\dagger}\right| J\right\rangle \\
& =\frac{1}{2} \sum_{\substack{j, k=1 \\
j \neq k}}^{N}\left(\delta_{n_{j}, n} \delta_{n_{k}, l} \delta_{n_{j}, m} \delta_{n_{k}, p}+\delta_{n_{j}, n} \delta_{n_{k}, l} \delta_{n_{k}, m} \delta_{n_{j}, p}\right. \\
& \left.\quad+\delta_{n_{j}, l} \delta_{n_{k}, n} \delta_{n_{j}, m} \delta_{n_{k}, p}+\delta_{n_{j}, l} \delta_{n_{k}, n} \delta_{n_{k}, m} \delta_{n_{j}, p}\right) .
\end{aligned}
$$


The appropriate energy eigenvalue in this sector can thus be computed by attaching coefficients of $a_{n}^{\dagger} a_{l}^{\dagger} a_{m} a_{p}$ in $H_{\mathrm{BB}}$ to the matrix element structure in eqn. (2.23). The $O(1 / J)$ energy correction for this protected $\mathfrak{s u}(2)$ sector of symmetric-traceless $S^{5}$ open string states is thereby found, for completely unequal mode indices, to be:

$$
\delta E_{S^{5}}\left(\left\{n_{i}\right\}, N, J\right)=-\frac{1}{8 J} \sum_{\substack{j, k=1 \\ j \neq k}}^{N} \frac{n_{k}^{2}+n_{j}^{2} \lambda^{\prime} \omega_{n_{k}}^{2}}{\omega_{n_{j}} \omega_{n_{k}}}
$$

By expanding in small $\lambda^{\prime}$

$$
\begin{aligned}
\delta E_{S^{5}}\left(\left\{n_{i}\right\}, N, J\right)= & \frac{1}{J} \sum_{\substack{j, k=1 \\
j \neq k}}^{N}\left\{-\frac{1}{8}\left(n_{j}^{2}+n_{k}^{2}\right) \lambda^{\prime}+\frac{1}{64}\left(n_{j}^{4}+n_{k}^{4}\right)\left(\lambda^{\prime}\right)^{2}\right. \\
& \left.-\frac{1}{1024}\left(3 n_{j}^{6}+n_{j}^{4} n_{k}^{2}+n_{j}^{2} n_{k}^{4}+3 n_{k}^{6}\right)\left(\lambda^{\prime}\right)^{3}+O\left(\left(\lambda^{\prime}\right)^{4}\right)\right\}
\end{aligned}
$$

it is easy to see that the spectrum for these states exhibits the expected property that at $m^{\text {th }}$ order in the expansion the energy scales with $2 m$ factors of the mode numbers $\left\{n_{i}\right\}$.

We can extend this result to the most general set of states in this sector, formed by a set of $a_{n}^{\dagger}$ creation oscillators grouped into $M$ subsets, with all mode indices equal within these subsets:

$$
\frac{\left(a_{n_{1}}^{\dagger}\right)^{N_{1}}}{\sqrt{N_{1} !}} \frac{\left(a_{n_{2}}^{\dagger}\right)^{N_{2}}}{\sqrt{N_{2} !}} \cdots \frac{\left(a_{n_{M}}^{\dagger}\right)^{N_{M}}}{\sqrt{N_{M} !}}|J\rangle
$$

The $j^{\text {th }}$ subset contains $N_{j}$ oscillators with mode index $n_{j}$, and the total impurity number is $N$, such that

$$
\sum_{j=1}^{M} N_{j}=N
$$

The matrix element of the oscillator structure $a_{n}^{\dagger} a_{l}^{\dagger} a_{m} a_{p}$ between these states is

$$
\begin{aligned}
&\langle J| \frac{\left(a_{n_{1}}\right)^{N_{n_{1}}}}{\sqrt{N_{n_{1}} !}} \cdots \frac{\left(a_{n_{M}}\right)^{N_{n_{M}}}}{\sqrt{N_{n_{M}} !}}\left(a_{n}^{\dagger} a_{l}^{\dagger} a_{m} a_{p}\right) \frac{\left(a_{n_{1}}^{\dagger}\right)^{N_{n_{1}}}}{\sqrt{N_{n_{1}} !}} \cdots \frac{\left(a_{n_{M}}^{\dagger}\right)^{N_{n_{M}}}}{\sqrt{N_{n_{M}} !}}|J\rangle \\
&=\sum_{j=1}^{M} N_{n_{j}}\left(N_{n_{j}}-1\right) \delta_{n, n_{j}} \delta_{l, n_{j}} \delta_{m, n_{j}} \delta_{p, n_{j}}+\frac{1}{2} \sum_{\substack{j, k=1 \\
j \neq k}}^{M} N_{n_{j}} N_{n_{k}}\left(\delta_{n, n_{k}} \delta_{l, n_{j}} \delta_{m, n_{k}} \delta_{p, n_{j}}\right. \\
&\left.+\delta_{n, n_{j}} \delta_{l, n_{k}} \delta_{m, n_{k}} \delta_{p, n_{j}}+\delta_{n, n_{k}} \delta_{l, n_{j}} \delta_{m, n_{j}} \delta_{p, n_{k}}+\delta_{n, n_{j}} \delta_{l, n_{k}} \delta_{m, n_{j}} \delta_{p, n_{k}}\right) .
\end{aligned}
$$


The energy shift at $O(1 / J)$ for completely general $N$-impurity open string states in the Dirichlet $\mathfrak{s u}(2)$ sector of the theory is therefore given by

$$
\begin{aligned}
\delta E_{S^{5}}\left(\left\{n_{i}\right\},\left\{N_{i}\right\}, M, J\right)= & -\frac{1}{8 J}\left\{\sum_{j=1}^{M} N_{j}\left(N_{j}-1\right) \frac{n_{j}^{2}\left(6+n_{j}^{2} \lambda^{\prime}\right)}{4 \omega_{n_{j}}^{2}}\right. \\
& \left.+\sum_{\substack{j, k=1 \\
j \neq k}}^{M} N_{j} N_{k} \frac{n_{k}^{2}+n_{j}^{2} \lambda^{\prime} \omega_{n_{k}}^{2}}{\omega_{n_{j}} \omega_{n_{k}}}\right\} .
\end{aligned}
$$

We see that the structure obtained for states with completely inequivalent mode indices in eqn. (2.24) appears in the second term above. This term can be thought of as the contribution to the energy from scattering among excitations with differing mode numbers; the first term represents scattering within subsets of equal mode numbers. For eventual comparison with corresponding quantities in the dual gauge theory, we expand eqn. (2.28) in small $\lambda^{\prime}$ :

$$
\begin{aligned}
\delta E_{S^{5}}\left(\left\{n_{i}\right\},\left\{N_{n_{i}}\right\}, M, J\right) & =\frac{1}{J} \sum_{k=1}^{M} N_{k}\left(N_{k}-1\right)\left[-\frac{3}{16} n_{k}^{2} \lambda^{\prime}+\frac{1}{64} n_{k}^{4}\left(\lambda^{\prime}\right)^{2}-\frac{1}{256} n_{k}^{6}\left(\lambda^{\prime}\right)^{3}\right] \\
& +\frac{1}{J} \sum_{\substack{k, j=1 \\
j \neq k}}^{M} N_{j} N_{k}\left[-\frac{1}{8}\left(n_{j}^{2}+n_{k}^{2}\right) \lambda^{\prime}+\frac{1}{64}\left(n_{j}^{4}+n_{k}^{4}\right)\left(\lambda^{\prime}\right)^{2}\right. \\
& \left.-\frac{1}{1024}\left(3 n_{j}^{6}+n_{j}^{4} n_{k}^{2}+n_{j}^{2} n_{k}^{4}+3 n_{k}^{6}\right)\left(\lambda^{\prime}\right)^{3}\right]+O\left(\left(\lambda^{\prime}\right)^{4}\right) .
\end{aligned}
$$

Within each of the $M$ subsectors of overlapping mode numbers (labeled by the indices $j$ and $k$ ) we again observe the desired result that the contribution to the energy scales at $m^{\text {th }}$ order in $\lambda^{\prime}$ in proportion to $n_{k}^{2 m}$.

\subsection{Neumann $S O(4)_{A d S}(\mathfrak{s l}(2))$ sector}

From table 2 we see that we can repeat the above calculation for the protected $\mathfrak{s l}(2)$ sector of symmetric-traceless bosonic open string states excited in the $A d S_{5}$ subspace. Analogous to eqn. (2.19) above, we can project onto this sector by forming the oscillators

$$
a_{n} \equiv \frac{1}{\sqrt{2}}\left(a_{n}^{1}+i a_{n}^{2}\right), \quad \bar{a}_{n} \equiv \frac{1}{\sqrt{2}}\left(a_{n}^{1}-i a_{n}^{2}\right)
$$

and restricting to unperturbed string states of the form

$$
a_{n_{1}}^{\dagger} a_{n_{2}}^{\dagger} \cdots a_{n_{N}}^{\dagger}|J\rangle
$$

Here we are free to project onto the $\left(z_{N}^{1}, z_{N}^{2}\right),\left(z_{N}^{2}, z_{N}^{3}\right)$ or $\left(z_{N}^{1}, z_{N}^{3}\right)$ planes, and we choose to exclude $z_{D}^{4}$ to avoid mixing boundary conditions. The states of interest carry Neumann boundary conditions, and the the mode expansion is given by

$$
x_{N}^{A}(\tau, \sigma)=\sum_{n=0}^{\infty} \frac{i}{\sqrt{\omega_{n}}} \cos \left(\frac{n \sigma}{2}\right)\left(a_{n}^{A} e^{-i \omega_{n} \tau}-a_{n}^{A \dagger} e^{i \omega_{n} \tau}\right) .
$$


(The equations of motion again require that $\omega_{n}$ satisfy the dispersion relation given in eqn. (2.18).)

The computation of the energy shift at $O(1 / J)$ for the $\mathfrak{s l}(2)$ sector of open string states follows in complete analogy with the $\mathfrak{s u}(2)$ sector described above. We form general matrix elements of the form given in eqn. (2.27) and attach coefficients of the Hamiltonian proportional to the oscillator structure $a_{n}^{\dagger} a_{l}^{\dagger} a_{m} a_{p}$, the only difference being that this sector of the interacting Hamiltonian is defined in terms of the worldsheet excitations in eqn. (2.31). Leaving out the computational details, we obtain the following energy shift for completely general open string states in the $\mathfrak{s l}(2)$ sector of the theory:

$$
\delta E_{A d S}\left(\left\{n_{i}\right\},\left\{N_{i}\right\}, M, J\right)=-\frac{1}{32 J}\left\{\sum_{k=1}^{M} N_{k}\left(N_{k}-1\right) \frac{n_{k}^{2}\left(2+n_{k}^{2} \lambda^{\prime}\right)}{\omega_{n_{k}}^{2}}+\sum_{\substack{j, k=1 \\ j \neq k}}^{M} N_{j} N_{k} \frac{n_{j}^{2} n_{k}^{2} \lambda^{\prime}}{\omega_{n_{j}} \omega_{n_{k}}}\right\} .
$$

For comparison with the gauge theory, we expand in $\lambda^{\prime}$ :

$$
\begin{aligned}
\delta E_{A d S}\left(\left\{n_{i}\right\},\left\{N_{i}\right\}, M, J\right)= & \frac{1}{J} \sum_{k=1}^{M} N_{k}\left(1-N_{k}\right)\left[\frac{1}{16} n_{k}^{2} \lambda^{\prime}+\frac{1}{64} n_{k}^{4}\left(\lambda^{\prime}\right)^{2}-\frac{1}{256} n_{k}^{6}\left(\lambda^{\prime}\right)^{3}\right] \\
& +\frac{1}{J} \sum_{\substack{j, k=1 \\
j \neq k}}^{M} N_{j} N_{k}\left[-\frac{1}{32} n_{j}^{2} n_{k}^{2}\left(\lambda^{\prime}\right)^{2}+\frac{1}{256} n_{j}^{2} n_{k}^{2}\left(n_{j}^{2}+n_{k}^{2}\right)\left(\lambda^{\prime}\right)^{3}\right] \\
& +O\left(\left(\lambda^{\prime}\right)^{4}\right) .
\end{aligned}
$$

The result again breaks up into contributions from interactions among worldsheet excitations with both equal and inequivalent mode numbers. There are also no contributions from zero-mode fluctuations: this is an important consistency check, since such corrections are prohibited in general [18,19]. One may also expand in small $\lambda^{\prime}$ to see that the scaling with mode numbers follows the same pattern demonstrated in eqns. (2.24) and (2.28) above.

\section{Open string Bethe equations}

There has recently been a great deal of work exploring the existence and role of integrable structures in both type IIB string theory on $A d S_{5} \times S^{5}$ and $\mathcal{N}=4$ SYM theory. It was originally noticed, first for the bosonic theory 44 and later for the full Green-Schwarz action [45], that the classical coset sigma model of the string on $A d S_{5} \times S^{5}$ possesses an infinite set of mutually commuting conserved charges. In [46], Arutyunov and Staudacher used a set of Bäcklund transformations to construct a generating function for these charges and matched their results to a corresponding generating function computed in the dual gauge theory. In 47], the Riemann-Hilbert problem for the classical finite-gap solutions of the sigma model, restricted to an $S^{2} \subset S^{5}$ subspace, was shown to be equivalent to the classical limit of the corresponding gauge theory Bethe equations. This result was extended to include 
the full sigma model in [48 51]. This was accompanied by [52, in which Berkovits used a pure-spinor formalism to argue that that the tower of commuting conserved charges persists in the quantum theory (see also [23] for an exploration of these charges in the quantum theory near the BMN limit). In [53], Arutyunov and Frolov were able to construct a Lax representation of the classical bosonic string Hamiltonian in a specific gauge; this result was extended by Alday, Arutyunov and Tseytlin to the gauge-fixed physical superstring in [54. (The approach to studying higher conserved charges using monodromy matrices was related to earlier investigations involving Bäcklund transformations in [55].) Finally, we note that studies of integrability for semiclassical rotating strings were extended beyond the planar limit in [56, 57].

On the gauge theory side, the presence of integrable structures was originally pointed out by Minahan and Zarembo [34], who showed that the action of the SYM dilatation operator on single-trace operators in a protected $S O(6)$-invariant sector of the theory can be mapped to that of an integrable Hamiltonian acting on a closed Heisenberg spin chain. The eigenvalues of the Hamiltonian, which are identified with corresponding operator dimensions, may thereby be obtained by solving a system of Bethe equations. Since we aim to formulate and solve a set of Bethe equations for the open string states examined in section 2, we will briefly review how this procedure is applied to the $\mathfrak{s u}(2)$ sector of $\mathcal{N}=4$ SYM theory at one-loop order in $\lambda$. (We will return to the higher-loop treatment of the gauge theory in section 4 below.)

In the planar limit, single-trace operators in the $\mathfrak{s u}(2)$ sector are built from complex scalars, typically denoted by $Z$ and $\phi$. The $Z$ fields are charged under the $U(1)_{R}$ component of the $S O(6) \cong U(1)_{R} \times S O(4)$ decomposition of the full $S U(4) R$-symmetry group, while the $\phi$ fields carry zero $R$-charge and act as impurity insertions. We obtain a basis of length- $L$ operators by forming field monomials that carry $N$ impurities and total $R$-charge equal to $R=L-N$ :

$$
\operatorname{tr}\left(\phi^{N} Z^{L-N}\right), \quad \operatorname{tr}\left(\phi^{N-1} Z \phi Z^{L-N-1}\right), \quad \operatorname{tr}\left(\phi^{N-2} Z \phi^{2} Z^{L-N-1}\right), \quad \ldots
$$

Unlike the $\mathfrak{s o}(6)$ sector originally studied by Minahan and Zarembo, which is protected from mixing at one-loop order in $\lambda$, the $\mathfrak{s u}(2)$ sector is protected at all orders in perturbation theory. ${ }^{3}$ There is a separate spin chain Hamiltonian $H_{\mathfrak{s u}(2)}^{(2 n)}$ appropriate for each order in the $\lambda$ expansion:

$$
H_{\mathfrak{s u}(2)}=N+\sum_{n} g^{2 n} H_{\mathfrak{s u}(2)}^{(2 n)}
$$

where $g^{2} \equiv \lambda / 8 \pi^{2}$. The loop expansion determines the generic form of the $n^{\text {th }}$-order Hamiltonian, since $H_{\mathfrak{s u}(2)}^{(2 n)}$ contains interactions that exchange fields separated by at most $n$ lattice

\footnotetext{
${ }^{3}$ In other words, the operators in eqn. (3.1) will not mix with other operators in the theory, and the anomalous dimension matrix can be diagonalized on this basis (at leading order in the large- $N_{c}$ expansion). We note, however, that a recent study by Minahan [58] indicates that mixing might occur in the $\mathfrak{s u}(2)$ sector in the strong coupling regime.
} 
sites. At one-loop order, $H_{\mathfrak{s u}(2)}^{(2)}$ is given explicitly by

$$
H_{\mathfrak{s u}(2)}^{(2)}=2 \sum_{k=1}^{N}\left(1-P_{k, k+1}\right)
$$

where $P_{k, k+1}$ exchanges fields on the $k^{\text {th }}$ and $(k+1)^{\text {th }}$ lattice sites. (The two- and three-loop extensions are given in [35 37.) The eigenvalues of this Hamiltonian can be obtained by solving the following Bethe equation

$$
e^{i p_{k} L}=\prod_{\substack{j=1 \\ j \neq k}}^{N} S\left(p_{k}, p_{j}\right)
$$

where the closed-chain $\mathfrak{s u}(2)$ scattering matrix $S\left(p_{k}, p_{j}\right)$ is given by

$$
S\left(p_{k}, p_{j}\right)=\frac{u_{k}-u_{j}+i}{u_{k}-u_{j}-i}
$$

The product on the right hand side of eqn. (3.4) runs over all impurity excitations, excluding the $k^{\text {th }}$ impurity, and the Bethe roots $u_{k}$ encode the pseudomomenta $p_{k}$ of lattice excitations via the relation

$$
u_{k}=\frac{1}{2} \cot \frac{p_{k}}{2}
$$

The form of (3.4) can be understood intuitively: the exponent on the left-hand-side represents the phase acquired by a pseudoparticle excitation as it is transported once around the lattice, and the equation states that this phase must be equal to that which is acquired by scattering the excitation off of every other impurity as is passes around the chain. The $S$ matrix interpretation of such equations was recently studied in detail in [59], where it was emphasized that the form of scattering matrices is tightly restricted by the constraints of integrability (scattering terms must be factorizable into pieces that encode at most twobody interactions; for related results relying on a virial expansion of the multi-loop spin chain Hamiltonians in $\mathcal{N}=4$ SYM theory, see [60]). For gauge-traced operators, which map to spin chain systems with periodic boundary conditions (i.e., closed spin chains), eqn. (3.4) is supplemented by the condition

$$
1=\prod_{k=1}^{N}\left(\frac{u_{k}+i / 2}{u_{k}-i / 2}\right)
$$

which enforces momentum conservation on the lattice and corresponds to the usual level matching condition in the dual sector of closed IIB string states in $A d S_{5} \times S^{5}$. (In other words, the set of mode numbers $\left\{n_{k}\right\}$ of physical states on the lattice are required by eqn. (3.7) to satisfy $\sum_{k} n_{k}=0$.) 
The Hamiltonian of this system appears as one in an infinite tower of conserved charges in the theory which, as noted above, is typical of integrable systems. The entire infinite set of charges is diagonalized by the Bethe ansatz in eqns. (3.4. 3.7), and the eigenvalues associated with each charge can be expressed in terms of the Bethe roots $u_{k}$. Operator anomalous dimensions at $O(\lambda)$ in this sector of the gauge theory are thus identified with the energy eigenvalues of the $\mathfrak{s u}(2)$ one-loop spin chain:

$$
E_{\mathfrak{s u}(2)}\left(\left\{n_{i}\right\}\right)=\frac{\lambda}{8 \pi^{2}} \sum_{k=1}^{N} \frac{1}{u_{k}^{2}+1 / 4} .
$$

The task of computing anomalous dimensions in this sector of $\mathcal{N}=4 \mathrm{SYM}$ theory is thereby reduced to that of solving eqns. (3.4, 3.7) for the Bethe roots $u_{k}$.

This application of the Bethe ansatz was extended to the full theory at one-loop order by Beisert and Staudacher in [61, where the authors formulated a set of Bethe equations for the full set of $\mathfrak{p s u}(2,2 \mid 4)$ fields in the theory. This methodology was extended beyond one-loop order in $\lambda$ in [33, where Serban and Staudacher used an Inozemtsev spin chain [62, 63] to capture higher-loop effects. (Such systems are referred to as long-range spin chains, because the Hamiltonian can mix spins that are not on neighboring lattice sites.) The energies (anomalous dimensions) admitted by the Inozemtsev system, however, do not conform to the requirements of BMN scaling at all orders in perturbation theory (i.e., at $O\left(\lambda^{n}\right)$, anomalous dimensions should scale at finite $R$-charge as $1 / R^{2 n}$ or, in the language of the string angular momentum, as $\left.1 / J^{2 n}\right)$. Beisert, Dippel and Staudacher were subsequently able to formulate a long-range Bethe ansatz that met the constraints of BMN scaling and correctly reproduced $\mathfrak{s u}(2)$ anomalous dimensions to three-loop order in $\lambda[31$.

Higher-loop physics in the $\mathfrak{s u}(2)$ sector of the gauge theory is captured by the following generalized $S$ matrix:

$$
S_{\mathfrak{s u}(2)}\left(p_{k}, p_{j}\right)=\frac{\phi\left(p_{k}\right)-\phi\left(p_{j}\right)+i}{\phi\left(p_{k}\right)-\phi\left(p_{j}\right)-i}
$$

where the functions $\phi\left(p_{k}\right)$ are given by

$$
\phi\left(p_{k}\right) \equiv \frac{1}{2} \cot \left(\frac{p_{k}}{2}\right) \sqrt{1+8 g^{2} \sin ^{2}\left(\frac{p_{k}}{2}\right)} .
$$

(We note, however, that this scattering matrix is derived asymptotically and, strictly speaking, is only valid for long spin chains.) Multi-loop operator dimensions are identified with the energies

$$
E_{\mathfrak{s u}(2)}\left(\left\{n_{i}\right\}, N, R\right)=L+g^{2} \sum_{k=1}^{N} q_{2}\left(p_{k}\right)
$$

where the functions $q_{r}\left(p_{k}\right)$ are defined as

$$
q_{r}\left(p_{k}\right) \equiv \frac{1}{g^{r-1}} \frac{2 \sin \left(\frac{p_{k}}{2}(r-1)\right)}{r-1}\left(\frac{\sqrt{1+8 g^{2} \sin ^{2}\left(\frac{p_{k}}{2}\right)}-1}{2 g \sin \left(\frac{p_{k}}{2}\right)}\right)^{r-1}
$$


As described in the introduction, these formulas lead to a general disagreement with string theory at three-loop order in $\lambda$ and beyond. The anomalous dimensions implied by the above scattering matrix disagree in this respect for all physical $N$-impurity states with the corresponding near-pp-wave energy spectrum of the closed string theory on $A d S_{5} \times S^{5}$ [18- 20 , 22. In a remarkable paper by Arutyunov, Frolov and Staudacher [64, the authors discovered a modification to the long-range gauge theory $S$ matrix in eqn. (3.9) that yields predictions consistent with the near-pp-wave energy spectrum of the string theory, and exhibits the correct $\lambda^{1 / 4}$ scaling behavior at strong coupling [3]. They accomplished this by including an additional phase contribution $\Phi\left(p_{k}, p_{j}\right)$ to the $\mathfrak{s u}(2)$ scattering matrix in eqn. (3.9). The resulting quantum string scattering matrix $S_{S^{5}}^{\mathrm{IIB}}$ takes the form

$$
S_{S^{5}}^{\mathrm{IIB}}\left(p_{k}, p_{j}\right)=S_{\mathfrak{s u}(2)}\left(p_{k}, p_{j}\right) \Phi\left(p_{k}, p_{j}\right),
$$

where $\Phi\left(p_{k}, p_{j}\right)$ is given by

$$
\Phi\left(p_{k}, p_{j}\right) \equiv \exp \left[2 i \sum_{r=0}^{\infty}\left(\frac{g^{2}}{2}\right)^{r+2}\left(q_{r+2}\left(p_{k}\right) q_{r+3}\left(p_{j}\right)-q_{r+3}\left(p_{k}\right) q_{r+2}\left(p_{j}\right)\right)\right] .
$$

It was demonstrated in 22 that the energies implied by the corresponding quantum string Bethe equations perfectly match energy spectra derived directly from the string theory for general closed string states in the $\mathfrak{s u}(2)$ sector (in the near-pp-wave limit). Since the energy spectra contain rather specialized structures (as we have already seen in the case of the open string states in section 21), this is a non-trivial result: it stands as evidence that the quantum string theory is integrable, at least in this limit.

In what follows we apply similar methods to the $\mathfrak{s u}(2)$ and $\mathfrak{s l}(2)$ sectors of the bosonic open string states studied above. Inspired by the Bethe ansatz methodology presented at one-loop order for the matching of open string energies with the dimensions of corresponding gauge theory operators in [5] interacting Hamiltonian $H_{\mathrm{BB}}$ on completely general open string states.

\subsection{Dirichlet $S O(4)_{S^{5}}(\mathfrak{s u}(2))$ ansatz}

On the open spin chain, pseudoparticle excitations (or magnons) pass from one end of the chain, scatter off of the boundary and return to the starting point. Each transition across the length of the chain contributes a factor of $\exp (i p L)$ to the phase (the effect on the phase should be additive, so the momentum is understood to be oriented in the positive direction after it scatters from the boundary). The total phase acquired in this process must be equal to that generated by scattering from each impurity on the chain as it travels to the boundary and back (after scattering from the boundary, the relative momenta of the other impurities on the chain acquire an overall sign change). We use this simple picture to propose a long-range 
Bethe ansatz for the $\mathfrak{s u}(2)$ Dirichlet sector of bosonic open string states: ${ }^{4}$

$$
\begin{aligned}
e^{2 i p_{k} L} & =\prod_{\substack{j=1 \\
j \neq k}}^{N} S_{S^{5}}^{\mathrm{IIB}}\left(p_{k}, p_{j}\right) S_{S^{5}}^{\mathrm{IIB}}\left(p_{k},-p_{j}\right) \\
& =\prod_{\substack{j=1 \\
j \neq k}}^{N} S_{\mathfrak{s u}(2)}\left(p_{k}, p_{j}\right) S_{\mathfrak{s u}(2)}\left(p_{k},-p_{j}\right) \Phi\left(p_{k}, p_{j}\right) \Phi\left(p_{k},-p_{j}\right) .
\end{aligned}
$$

The long-range scattering matrices $S_{\mathfrak{s u}(2)}$ are defined in eqn. (3.9) above, and the phase corrections $\Phi\left(p_{k}, p_{j}\right)$ for the string are given in eqn. (3.14). We might have included some prefactor dependent on $p_{k}$ to capture phase contributions due to scattering from the boundary itself. We will see, however, that in this sector such contributions turn out to be trivial (this situation changes when we examine the $\mathfrak{s l}(2)$ sector below). We also note that for open spin chains there is no momentum condition that enforces level matching among the mode numbers (just as there is no level matching condition for the open string states studied in section 21).

To simplify the presentation, and to review a general strategy for solving eqn. (3.15) [34,64, we will first compute energy spectra in the near-pp-wave limit for length- $L$ states of the open chain whose impurities do not form bound states. These are the bosonic, symmetrictraceless open string states given in eqn. (2.15) above, with $\left(n_{1} \neq n_{2} \neq \cdots \neq n_{N}\right)$. We must solve the Bethe ansatz in eqn. (3.15) order by order in the large- $J$ expansion and extract contributions to the energy spectrum at $O(1 / J)$. In terms of the string angular momentum $J$ and the total impurity number $N$, the lattice length $L$ for the corresponding integrable spin chain is

$$
L=J-1+N
$$

When we analyze the dual gauge theory in section 4 below we will see how this equation is determined. For the moment, however, we will proceed without further motivation.

A useful technique, as demonstrated in [34 and [64], for example, is to expand the excitation momenta according to

$$
p_{k}=\frac{\pi n_{k}}{J}+\frac{p_{k}^{(1)}}{J^{3 / 2}}+\frac{p_{k}^{(2)}}{J^{2}}+O\left(1 / J^{5 / 2}\right),
$$

and solve eqn. (3.15) for $p_{k}^{(1)}, p_{k}^{(2)}$, etc. The leading contribution carries the integer mode number $n_{k}$ of the pseudomomentum $p_{k}$. (To compute energy eigenvalues to $O(1 / J)$, we will not need to find contributions to $p_{k}$ beyond $O\left(1 / J^{2}\right)$.) We first note that the coefficient $p_{k}^{(1)}$ is nonzero only in the presence of bound momentum states, where some set of $p_{k}$ share the

\footnotetext{
${ }^{4}$ This ansatz is motivated by gauge theory proposals at one-loop order in $\lambda[6]$. We note, however, that the specific equation given in [7] appears with a sign discrepancy.
} 
same mode number $n_{k}$. In the absence of bound states, the contribution to eqn. (3.15) from the phase factors $\Phi\left(p_{k}, p_{j}\right)$ is

$$
\Phi\left(p_{k}, p_{j}\right) \Phi\left(p_{k},-p_{j}\right)=1-\frac{2 i n_{k} \pi}{J p_{-}\left(n_{k}^{2}-n_{j}^{2}\right)}\left[n_{k}^{2}\left(\omega_{n_{j}}-p_{-}\right)-n_{j}^{2}\left(\omega_{n_{k}}-p_{-}\right)\right]+O\left(1 / J^{2}\right) .
$$

The Bethe equation in (3.15) is then satisfied by

$$
p_{k}^{(2)}=-n_{k} \pi(N-1)-\sum_{\substack{j=1 \\ j \neq k}}^{N} \frac{n_{k} \pi}{p_{-}\left(n_{k}^{2}-n_{j}^{2}\right)}\left[n_{k}^{2}\left(\omega_{n_{j}}-p_{-}\right)+n_{j}^{2}\left(\omega_{n_{k}}+p_{-}\right)\right] .
$$

From eqn. (3.11) above, we therefore find the following contribution to the energy spectrum at $O(1 / J)$ :

$$
\delta E\left(\left\{n_{i}\right\}, N, J\right)=\frac{1}{J} \sum_{k=1}^{N} \frac{n_{k} p_{k}^{(2)}}{4 \pi p_{-} \omega_{n_{k}}}=\frac{1}{J} \sum_{\substack{j, k=1 \\ j \neq k}}^{N} \frac{n_{k}^{2}+n_{j}^{2} \lambda^{\prime} \omega_{n_{k}}^{2}}{\omega_{n_{j}} \omega_{n_{k}}}
$$

This matches the corresponding energy correction for string states with no overlapping mode numbers computed directly from the string lightcone Hamiltonian in eqn. (2.24).

Solving the Bethe equation in the presence of bound states is slightly more complicated. To align with conventions in the literature we will adopt a notation similar to that presented in [64, wherein the complete set of $N$ mode numbers associated with each impurity excitation is divided into $M$ subsets of equal mode numbers labeled by the index $k$ :

$$
\{\underbrace{\left\{n_{1}, n_{1}, \ldots, n_{1}\right\}}_{k=1}, \underbrace{\left\{n_{2}, n_{2}, \ldots, n_{2}\right\}}_{k=2}, \ldots, \underbrace{\left\{n_{M}, n_{M}, \ldots, n_{M}\right\}}_{k=M}\} .
$$

The $k^{\text {th }}$ subset contains $N_{k}$ total numbers $n_{k}$, which are individually labeled by an index $m_{k} \in 1, \ldots, N_{k}$. The Bethe ansatz therefore takes the general form

$$
\begin{aligned}
\exp \left(2 i p_{k, m_{k}} L\right)= & \prod_{\substack{l_{k}=1 \\
l_{k} \neq m_{k}}}^{N_{k}} S_{S^{5}}^{\mathrm{IIB}}\left(p_{k, m_{k}}, p_{k, l_{k}}\right) S_{S^{5}}^{\mathrm{IIB}}\left(p_{k, m_{k}},-p_{k, l_{k}}\right) \\
& \times \prod_{\substack{j=1 \\
j \neq k}}^{M} \prod_{m_{j}=1}^{N_{j}} S_{S^{5}}^{\mathrm{IIB}}\left(p_{k, m_{k}}, p_{j, m_{j}}\right) S_{S^{5}}^{\mathrm{IIB}}\left(p_{k, m_{k}},-p_{j, m_{j}}\right),
\end{aligned}
$$

where the momenta now carry the labels $k$ and $m_{k}$, and are expanded in large $J$ according to

$$
p_{k, m_{k}}=\frac{\pi n_{k}}{J}+\frac{p_{k, m_{k}}^{(1)}}{J^{3 / 2}}+\frac{p_{k, m_{k}}^{(2)}}{J^{2}}+O\left(1 / J^{5 / 2}\right)
$$


Within each bound state, the contribution from factors of the phase correction $\Phi\left(p_{k, m_{k}}, p_{k, l_{k}}\right)$ is

$$
\Phi\left(p_{k, m_{k}}, p_{k, l_{k}}\right) \Phi\left(p_{k, m_{k}},-p_{k, l_{k}}\right)=1-\frac{i n_{k} \pi}{4 p_{-} \omega_{n_{k}} J}\left(8 p_{-}^{2}+n_{k}^{2}-8 p_{-} \omega_{n_{k}}\right)+O\left(1 / J^{2}\right)
$$

and the coefficient $p_{k, m_{k}}^{(1)}$ is now nonzero in the presence of bound states:

$$
p_{k, m_{k}}^{(1)}=-\frac{n_{k}^{2} \pi^{2} \omega_{n_{k}}}{p_{-}} \sum_{\substack{l_{k}=1 \\ l_{k} \neq m_{k}}}^{N_{k}} \frac{1}{p_{k, m_{k}}^{(1)}-p_{k, l_{k}}^{(1)}} .
$$

As for the closed-chain case in [64], we have found a generalized Stieltjes problem [65] which is solved by setting

$$
\left(p_{k, m_{k}}^{(1)}\right)^{2}=-\frac{n_{k}^{2} \pi^{2} \omega_{n_{k}}}{2 p_{-}} u_{N_{k}, m_{k}}^{2}
$$

where $u_{N_{k}, m_{k}}$ are the roots of the Hermite polynomials $Q_{N_{k}}(u)$ satisfying $Q^{\prime \prime}(u)-u Q^{\prime}(u)+$ $N_{k} Q(u)=0$.

At the next subleading order we find that $p_{k, m_{k}}^{(2)}$ is given by

$$
\begin{aligned}
p_{k, m_{k}}^{(2)}= & (1-N) \pi n_{k}-\frac{1}{2} \sum_{\substack{l_{k}=1 \\
l_{k} \neq m_{k}}}^{N_{k}}\left[p_{k, m_{k}}^{(2)}-p_{k, l_{k}}^{(2)}+n_{k} \pi\left(\frac{\omega_{n_{k}}}{p_{-}}-2\right)\right] \\
& -\sum_{\substack{j=1 \\
j \neq k}}^{M} \frac{n_{k} \pi N_{j}}{p_{-}\left(n_{k}^{2}-n_{j}^{2}\right)}\left[n_{k}^{2}\left(\omega_{n_{j}}-p_{-}\right)+n_{j}^{2}\left(\omega_{n_{k}}+p_{-}\right)\right] .
\end{aligned}
$$

We can simplify matters by noting that when we expand the energy formula in eqn. (3.11) to the order of interest, the correction at $O(1 / J)$ involves a sum over the index $m_{k}$ :

$$
E\left(\left\{n_{k}\right\}\right)=J+\sum_{k=1}^{M} \frac{2 N_{k} \omega_{n_{k}}}{p_{-}}+\frac{1}{p_{-} J} \sum_{k=1}^{M} \sum_{m_{k}=1}^{N_{k}} \frac{1}{8 \pi^{2} \omega_{n_{k}}^{3}}\left[p_{-}^{2}\left(p_{k, m_{k}}^{(1)}\right)^{2}+2 \pi n_{k} \omega_{n_{k}}^{2} p_{k, m_{k}}^{(2)}\right] .
$$

Under this sum the roots $u_{N_{k}, m_{k}}$ of the Hermite polynomials $Q_{N_{k}}(u)$ satisfy

$$
\sum_{m_{k}=1}^{N_{k}} u_{N_{k}, m_{k}}^{2}=N_{k}\left(N_{k}-1\right)
$$

Furthermore, contributions to the right hand side of eqn. (3.27) involving $p_{k, m_{k}}^{(2)}$ and $p_{k, l_{k}}^{(2)}$ will cancel:

$$
\sum_{m_{k}=1}^{N_{k}} \frac{n_{k}}{4 \pi \omega_{n_{k}}} p_{k, m_{k}}^{(2)}=\frac{n_{k}^{2}}{8 p_{-}} N_{k}\left(1-N_{k}\right)+\sum_{\substack{j=1 \\ j \neq k}}^{N} \frac{N_{j} N_{k}}{8 p_{-}\left(n_{j}^{2}-n_{k}^{2}\right)}\left(n_{k}^{4} \frac{\omega_{n_{j}}}{\omega_{n_{k}}}-n_{j}^{4} \frac{\omega_{n_{k}}}{\omega_{n_{j}}}\right) .
$$


We therefore arrive at a final expression for the correction to the energy spectrum at $O(1 / J)$ in the near-pp-wave limit:

$$
\begin{aligned}
\delta E_{S^{5}}\left(\left\{n_{i}\right\},\left\{N_{i}\right\}, M, J\right)= & -\frac{1}{8 J}\left\{\sum_{k=1}^{M} N_{k}\left(N_{k}-1\right) \frac{n_{k}^{2}\left(6+n_{k}^{2} \lambda^{\prime}\right)}{4 \omega_{n_{k}}^{2}}\right. \\
& \left.+\sum_{\substack{j, k=1 \\
j \neq k}}^{M} N_{j} N_{k} \frac{n_{k}^{2}+n_{j}^{2} \lambda^{\prime} \omega_{n_{k}}^{2}}{\omega_{n_{j}} \omega_{n_{k}}}\right\} .
\end{aligned}
$$

This matches the general $\mathfrak{s u}(2)$ string theory prediction in eqn. (2.28).

\subsection{Neumann $S O(4)_{A d S}(\mathfrak{s l}(2))$ ansatz}

We can extend this analysis to the protected $\mathfrak{s l}(2)$ sector of bosonic open string states. The open-string Bethe equation we propose is based on the long-range quantum string ansatz presented in [59] for the corresponding sector of $\mathfrak{s l}(2)$ closed-string states:

$$
e^{i p_{k} L}=\prod_{\substack{j=1 \\ j \neq k}}^{N} S_{A d S}^{\mathrm{IIB}}\left(p_{k}, p_{j}\right)
$$

where the scattering matrix $S_{A d S}^{\mathrm{IIB}}$ is given by

$$
S_{A d S}^{\mathrm{IIB}}\left(p_{k}, p_{j}\right) \equiv \frac{\phi\left(p_{k}\right)-\phi\left(p_{j}\right)-i}{\phi\left(p_{k}\right)-\phi\left(p_{j}\right)+i} \Psi\left(p_{k}, p_{p_{j}}\right)
$$

and the phase $\Psi\left(p_{k}, p_{p_{j}}\right)$ is defined as

$$
\Psi\left(p_{k}, p_{j}\right) \equiv \exp \left[-2 i \sum_{r=0}^{\infty}\left(\frac{g^{2}}{2}\right)^{r+1}\left(q_{r+1}\left(p_{k}\right) q_{r+2}\left(p_{j}\right)-q_{r+2}\left(p_{k}\right) q_{r+1}\left(p_{j}\right)\right)\right]
$$

This ansatz was derived in [59] using general considerations of integrability and of the structure of the near-pp-wave energy spectrum computed from the string theory [22]. The lattice length $L$ obeys

$$
L=J-1
$$

This formula is again based on gauge theory considerations, and will be discussed in section 4 below.

Using this scattering matrix, we propose the following general Bethe ansatz (allowing for confluent mode numbers) for the $\mathfrak{s l}(2)$ sector of open string states fluctuating in the 
Neumann directions of the $A d S_{5}$ subspace:

$$
\begin{aligned}
\exp \left(2 i p_{k, m_{k}} L\right)= & e^{-2 i p_{k, m_{k}}} \prod_{\substack{l_{k}=1 \\
l_{k} \neq m_{k}}}^{N_{k}} S_{A d S}^{\mathrm{IIB}}\left(p_{k, m_{k}}, p_{k, l_{k}}\right) S_{A d S}^{\mathrm{IIB}}\left(p_{k, m_{k}},-p_{k, l_{k}}\right) \\
& \times \prod_{\substack{j=1 \\
j \neq k}}^{M} \prod_{m_{j}=1}^{N_{j}} S_{A d S}^{\mathrm{IIB}}\left(p_{k, m_{k}}, p_{j, m_{j}}\right) S_{A d S}^{\mathrm{IIB}}\left(p_{k, m_{k}},-p_{j, m_{j}}\right) .
\end{aligned}
$$

The factor $e^{-2 i p_{k, m_{k}}}$ on the right-hand side is a phase contribution interpreted as being associated with scattering from the boundary. Note also that in the small- $\lambda^{\prime}$ expansion contributions to the Bethe equation from $\Psi\left(p_{k}, p_{j}\right)$ enter at a lower order than those for $\Phi\left(p_{k}, p_{j}\right)$ in the $\mathfrak{s u}(2)$ case considered above.

Expanding the pseudoparticle momenta according to eqn. (3.23), we find the following equation at first subleading order:

$$
p_{k, m_{k}}^{(1)}=\frac{n_{k}^{2} \pi^{2} \omega_{n_{k}}}{p_{-}} \sum_{\substack{l_{k}=1 \\ l_{k} \neq m_{k}}}^{N_{k}} \frac{1}{p_{k, m_{k}}^{(1)}-p_{k, l_{k}}^{(1)}} .
$$

This differs by an overall sign from the corresponding $\mathfrak{s u}(2)$ equation computed in (3.25) above. At next order in the $1 / J$ expansion we find

$$
p_{k, m_{k}}^{(2)}=\frac{1}{2} \sum_{\substack{l_{k}=1 \\ l_{k} \neq m_{k}}}^{N_{k}}\left(p_{k, l_{k}}^{(2)}-p_{k, m_{k}}^{(2)}-\frac{n_{k} \pi}{p_{-}} \omega_{n_{k}}\right)+\sum_{\substack{j=1 \\ j \neq k}}^{M} N_{j} \frac{F_{1}\left(n_{j}, n_{k}\right)}{F_{2}\left(n_{j}, n_{k}\right)}
$$

where, for convenience, we have defined

$$
\begin{aligned}
& F_{1}\left(n_{j}, n_{k}\right) \equiv-n_{j}^{2} n_{k}^{2} \pi\left\{8+\left(n_{j}^{2}+n_{k}^{2}\right) \lambda^{\prime}\right. \\
&\left.-\sqrt{\lambda^{\prime}}\left[\left(8+n_{k}\left(n_{k}-n_{j}\right) \lambda^{\prime}\right) \omega_{n_{j}}+\omega_{n_{k}}\left(8+n_{j}\left(n_{j}-n_{k}\right) \lambda^{\prime}-8 \sqrt{\lambda^{\prime}} \omega_{n_{j}}\right)\right]\right\} \\
& F_{2}\left(n_{j}, n_{k}\right) \equiv\left(n_{j}^{2}-n_{k}^{2}\right)\left[-4+n_{j} n_{k} \lambda^{\prime}+4 \sqrt{\lambda^{\prime}}\left(\omega_{n_{k}}+\omega_{n_{j}}-\sqrt{\lambda^{\prime}} \omega_{n_{k}} \omega_{n_{j}}\right)\right]
\end{aligned}
$$

Using the general expansion of the energy in eqn. (3.28) above, we arrive at the following formula for the $O(1 / J)$ energy shift in the near-pp-wave limit:

$$
\delta E_{A d S}\left(\left\{n_{i}\right\},\left\{N_{i}\right\}, M, J\right)=-\frac{1}{32 J}\left\{\sum_{k=1}^{M} N_{k}\left(N_{k}-1\right) \frac{n_{k}^{2}\left(2+n_{k}^{2} \lambda^{\prime}\right)}{\omega_{n_{k}}^{2}}+\sum_{\substack{j, k=1 \\ j \neq k}}^{M} N_{j} N_{k} \frac{n_{j}^{2} n_{k}^{2} \lambda^{\prime}}{\omega_{n_{j}} \omega_{n_{k}}}\right\} .
$$

This is precisely the energy expression computed for the $\mathfrak{s l}(2)$ sector of the string theory in eqn. (2.32) above. 


\section{SYM long-range Bethe equations}

As noted in section 3, a long-range Bethe ansatz for the protected sector of single-trace $\mathfrak{s u}(2)$ operators in $\mathcal{N}=4$ SYM theory was formulated in [31, and an analogous $\mathfrak{s l}(2)$ ansatz was given more recently in [59]. Building on our successful derivation of the openstring $\mathfrak{s u}(2)$ Bethe equations in (3.15, 3.22) based on corresponding equations formulated for closed string states, we can immediately write down long-range, open-chain Bethe ansatz equations for $\mathfrak{s u}(2)$ and $\mathfrak{s l}(2)$ sectors of the $\mathcal{N}=2$ defect conformal field theory dual to the corresponding open string states described in section 2 . The theory we are interested in describes a stack of $N_{c}$ coincident $D 3$-branes with one $D 5$-brane extended along three of the worldvolume dimensions of the $D 3$ stack (see table 1). This theory, studied in [7, 8, 39], contains a three-dimensional $\mathcal{N}=4 S U\left(N_{c}\right)$ hypermultiplet in addition to the bulk fourdimensional hypermultiplet. The $D 5$-brane defect preserves an $S O(3,2)$ subgroup of the conformal group and eight of the supersymmetries, though it breaks the $R$-symmetry from $S U(4)$ to $S U(2)_{H} \times S U(2)_{V}$. We can decompose the bulk $\mathcal{N}=4$ vector multiplet into a threedimensional $\mathcal{N}=4$ vector multiplet and a three-dimensional $\mathcal{N}=4$ adjoint hypermultiplet. The vector multiplet contains the following bosonic fields:

$$
A_{\mu}, \quad X^{1}, \quad X^{2}, \quad X^{3}, \quad D_{3} X^{I},
$$

with $\mu \in 0,1,2$ and $I \in 4,5,6$. The hypermultiplet contains the component of the gauge field normal to the defect, as well as the scalars $X^{4}, X^{5}, X^{6}$ and $D_{3} X^{A}$, with $A \in 1,2,3$. The three-dimensional $\mathcal{N}=4 S U\left(N_{c}\right)$ hypermultiplet contains a set of complex scalars $q^{m}$ (with $m \in 1,2$ ) which couple canonically to the gauge fields. On the string side we take the Penrose limit by boosting in the direction dual to the $(1,2)$ plane. The charge $J_{S U(2)_{H}}^{3}$ is identified with the string angular momentum $J$ (which we will refer to simply as the $R$-charge), and the fields of interest are thus charged under $S U(2)_{H}$ according to

$$
X^{1}, X^{2}, X^{3}(J=1), \quad X^{4}, X^{5}, X^{6}(J=0), \quad q^{m}, \tilde{q}^{m} \quad(J=1 / 2) .
$$

The Bethe ground state is taken to be

$$
\overline{q_{1}} Z \cdots Z q_{2}
$$

with $Z \equiv X^{1}+i X^{2}$. (The indices on the fields $q^{m}$ are lowered by Chan-Paton factors; see [] for further details.) $R$-charge impurities in the $\mathfrak{s u}(2)$ sector are denoted by $W \equiv X^{4}+i X^{5}$, and we form a basis of length- $L$ (i.e., operator monomials containing a total of $L$ fields), $N$-impurity operators given by

$$
\overline{q_{1}} W^{N} Z^{L-N-2} q_{2}, \quad \overline{q_{1}} W^{N-1} Z W Z^{L-N-3} q_{2}, \quad \overline{q_{1}} W^{N-2} Z W^{2} Z^{L-N-4} q_{2}, \quad \ldots
$$

This basis corresponds to the $\mathfrak{s u}(2)$ sector of open Dirichlet string states in the $S^{5}$ subspace. With the $R$-charge assignments given above, we now have the relation given in eqn. (3.16):

$$
L_{\mathfrak{s u}(2)}=J-1+N \text {. }
$$


The sector of $\mathfrak{s l}(2)$ open string states with Neumann boundary conditions is dual to operators with derivative insertions (with $D \equiv D_{1}+i D_{2}$ ):

$$
\overline{q_{1}} D^{N} Z^{L-N-2} q_{2}, \quad \overline{q_{1}} D^{N-1} Z D Z^{L-N-3} q_{2}, \quad \overline{q_{1}} D^{N-2} Z D^{2} Z^{L-N-4} q_{2}, \quad \ldots
$$

so that the lattice length of the corresponding $\mathfrak{s l}(2)$ open spin chain is

$$
L_{\mathfrak{s l}(2)}=J-1
$$

as given in eqn. (3.35). By mapping the dilatation generator to the Hamiltonian of an integrable open spin chain, Bethe ansatz equations for similar sectors of operators were derived at one-loop order in $\lambda$ in [6, [7].

\subsection{Long-range $\mathfrak{s u}(2)$ ansatz}

We will focus first on the closed-chain, long-range scattering matrix for the $\mathfrak{s u}(2)$ spin chain, derived in 31] and given above in eqn. (3.9). In terms of this $S$ matrix, we propose the following long-range, open-chain ansatz for this sector:

$$
e^{2 i p_{k} L}=\prod_{j \neq k}^{N} S_{\mathfrak{s u}(2)}\left(p_{k}, p_{j}\right) S_{\mathfrak{s u}(2)}\left(p_{k},-p_{j}\right) .
$$

As discussed in section 3, we could include a phase contribution due to scattering at the boundaries. Given what we have learned from the corresponding $\mathfrak{s u}(2)$ string Bethe equations, however, we expect that such contributions are trivial.

The full Bethe equation takes the form

$$
\begin{aligned}
\exp \left(2 i L p_{k, m_{k}}\right)= & \prod_{\substack{l_{k}=1 \\
l_{k} \neq m_{k}}}^{N_{k}} S_{\mathfrak{s u}(2)}\left(p_{k, m_{k}}, p_{k, l_{k}}\right) S_{\mathfrak{s u}(2)}\left(p_{k, m_{k}},-p_{k, l_{k}}\right) \\
& \times \prod_{\substack{j=1 \\
j \neq k}}^{M} \prod_{m_{j}=1}^{N_{j}} S_{\mathfrak{s u}(2)}\left(p_{k, m_{k}}, p_{j, m_{j}}\right) S_{\mathfrak{s u}(2)}\left(p_{k, m_{k}},-p_{j, m_{j}}\right) .
\end{aligned}
$$

Expanding pseudoparticle momenta in the usual fashion

$$
p_{k, m_{k}}=\frac{n_{k} \pi}{J}+\frac{p_{k, m_{k}}^{(1)}}{J^{3 / 2}}+\frac{p_{k, m_{k}}^{(2)}}{J}+O\left(1 / J^{3 / 2}\right),
$$

we find that eqn. (4.6) is satisfied to first subleading order within the $k^{\text {th }}$ subset of overlapping mode numbers for

$$
p_{k, m_{k}}^{(1)}=-\frac{n_{k}^{2} \pi^{2} \omega_{n_{k}}}{p_{-}} \sum_{\substack{l_{k}=1 \\ l_{k} \neq m_{k}}}^{N_{k}} \frac{1}{p_{k, m_{k}}^{(1)}-p_{k, l_{k}}^{(1)}}
$$


As in the case of the string Bethe ansatz above, this Stieltjes problem is solved by setting

$$
\left(p_{k, m_{k}}^{(1)}\right)^{2}=-\frac{\pi^{2} n_{k}^{2} \omega_{n_{k}}}{2 p_{-}} u_{N_{k}, m_{k}}^{2}
$$

which is identical to the string theory solution in eqn. (3.26) above.

At the next subleading order we find

$$
p_{k, m_{k}}^{(2)}=(1-N) \pi n_{k}-\frac{1}{2} \sum_{\substack{l_{k}=1 \\ l_{k} \neq m_{k}}}^{N_{k}}\left(p_{k, m_{k}}^{(2)}-p_{k, l_{k}}^{(2)}-\frac{p_{-} \pi n_{k}}{\omega_{n_{k}}}\right)+\sum_{\substack{j=1 \\ j \neq k}}^{M} N_{j} \frac{2 \pi n_{k} n_{j}^{2} \omega_{n_{k}}}{p_{-}\left(n_{j}^{2}-n_{k}^{2}\right)} .
$$

The contribution from $p_{k, m_{k}}^{(2)}$ to the $O(1 / J)$ energy correction again involves a sum over the index $m_{k}$, so that contributions to $\delta E$ from terms proportional to $p_{k, m_{k}}^{(2)}-p_{k, l_{k}}^{(2)}$ will cancel. Proceeding in the same fashion as before, we find a final expression for the energy spectrum at $O(1 / J)$ in the near-BMN limit:

$$
\begin{aligned}
\delta E_{\mathfrak{s u}(2)}\left(\left\{n_{i}\right\},\left\{N_{i}\right\}, M, J\right)= & \frac{1}{p_{-} J} \sum_{k=1}^{M} \frac{n_{k}^{2}}{16 \omega_{n_{k}}^{2}} N_{k}\left(N_{k}-1\right)\left(p_{-}-4 \omega_{n_{k}}\right) \\
& -\frac{1}{p_{-} J} \sum_{\substack{k, j=1 \\
j \neq k}}^{M} N_{j} N_{k} \frac{n_{k}^{2}}{4 \omega_{n_{k}}} .
\end{aligned}
$$

The energy formula again breaks neatly into contributions associated with scattering within and between bound momentum states of the spin chain. Expanding in small $\lambda^{\prime}$, we obtain

$$
\begin{aligned}
& \delta E_{\mathfrak{s u}(2)}\left(\left\{n_{i}\right\},\left\{N_{i}\right\}, M, J\right)= \frac{1}{J} \sum_{k=1}^{M} N_{k}\left(N_{k}-1\right)\left[-\frac{3}{16} n_{k}^{2} \lambda^{\prime}+\frac{1}{64} n_{k}^{4}\left(\lambda^{\prime}\right)^{2}-\frac{1}{512} n_{k}^{6}\left(\lambda^{\prime}\right)^{3}\right] \\
&+\frac{1}{J} \sum_{\substack{k, j=1 \\
j \neq k}}^{M} N_{j} N_{k}\left[-\frac{1}{8}\left(n_{j}^{2}+n_{k}^{2}\right) \lambda^{\prime}+\frac{1}{64}\left(n_{j}^{4}+n_{k}^{4}\right)\left(\lambda^{\prime}\right)^{2}\right. \\
&\left.-\frac{3}{1024}\left(n_{j}^{6}+n_{k}^{6}\right)\left(\lambda^{\prime}\right)^{3}\right]+O\left(\left(\lambda^{\prime}\right)^{4}\right) .
\end{aligned}
$$

Once again, we find perfect agreement with the corresponding $\mathfrak{s u}(2)$ string theory predictions in eqn. (2.29) at one- and two-loop order, but a disagreement at three loops and beyond!

\subsection{Long-range $\mathfrak{s l}(2)$ ansatz}

One might guess that the correct closed-chain $\mathcal{N}=4 \mathfrak{s l}(2)$ scattering matrix can be obtained from eqn. (3.33) simply by eliminating the phase contribution from $\Psi\left(p_{k}, p_{j}\right)$. From the structure of the phase factor $\Psi\left(p_{k}, p_{j}\right)$, we can immediately see that this would lead to a 
general mismatch at two-loop order and beyond. It turns out that this simple hypothesis is incorrect. The correct asymptotic gauge theory scattering matrix was derived in [59]:

$$
S_{\mathfrak{s l}(2)}\left(p_{k}, p_{j}\right) \equiv \frac{\phi\left(p_{k}\right)-\phi\left(p_{j}\right)-i}{\phi\left(p_{k}\right)-\phi\left(p_{j}\right)+i} e^{2 i \theta\left(p_{k}, p_{j}\right)} .
$$

The phase shift $\theta\left(p_{k}, p_{j}\right)$ was originally formulated in the computation of a long-range scattering matrix for the fermionic $\mathfrak{s u}(1 \mid 1)$ sector of $\mathcal{N}=4$ SYM via Staudacher's method of the perturbative asymptotic Bethe ansatz (see [59] for further details):

$$
S_{\mathfrak{s u}(1 \mid 1)}\left(p_{k}, p_{j}\right)=-e^{i \theta\left(p_{k}, p_{j}\right)} .
$$

The $\mathfrak{s l}(2) S$ matrix in eqn. (4.13) was then derived from this $\mathfrak{s u}(1 \mid 1)$ scattering matrix and the corresponding matrix in the $\mathfrak{s u}(2)$ sector (3.9) using the following remarkable relationship:

$$
S_{\mathfrak{s l}(2)}=S_{\mathfrak{s u}(1 \mid 1)} S_{\mathfrak{s u}(2)}^{-1} S_{\mathfrak{s u}(1 \mid 1)} .
$$

According to [59], this equation appears to hold for both the string theory and gauge theory. The phase $\theta\left(p_{k}, p_{j}\right)$ was calculated perturbatively in small $\lambda$ in [59], yielding:

$$
\begin{aligned}
\theta\left(p_{k}, p_{j}\right)= & {\left[2 g^{2} \sin ^{2} \frac{p_{k}}{2} \sin p_{j}-2 g^{4} \sin ^{4} \frac{p_{k}}{2} \sin 2 p_{j}+8 g^{4} \sin p_{k} \sin ^{2} \frac{p_{k}}{2} \sin ^{2} \frac{p_{j}}{2}\right] } \\
& -\left(p_{k} \leftrightharpoons p_{j}\right) .
\end{aligned}
$$

The expansion to this order $\left(O\left(\lambda^{2}\right)\right)$ makes the resulting scattering matrix (4.13) accurate up to and including three-loop order in $\lambda$; a higher-order expansion would require specific knowledge of certain four-loop vertices in the $\mathcal{N}=4$ gauge theory. ${ }^{5}$

In terms of the $\mathfrak{s l}(2) S$ matrix in eqn. (4.13), we arrive at the following long-range, open-chain Bethe ansatz for the $\mathfrak{s l}(2)$ sector (4.3) of the defect conformal field theory:

$$
\exp \left(2 i p_{k} L\right)=e^{-2 i p_{k}} \prod_{\substack{j=1 \\ j \neq k}}^{N} S_{\mathfrak{s l}(2)}\left(p_{k}, p_{j}\right) S_{\mathfrak{s l}(2)}\left(p_{k},-p_{j}\right) .
$$

The additional phase shift $e^{-2 i p_{k}}$ includes the effects of boundary scattering, analogous to the corresponding open string ansatz in eqn. (3.36). We can solve this equation in the nearBMN limit using the methods described in detail above. Omitting the computational details, we find the following general energy shift (for all possible mode-index distributions) in the near-BMN limit:

$$
\begin{aligned}
\delta E_{\mathfrak{s l}(2)}\left(\left\{n_{i}\right\},\left\{N_{i}\right\}, M, J\right)= & \frac{1}{J} \sum_{k=1}^{M} N_{k}\left(1-N_{k}\right)\left[\frac{1}{16} n_{k}^{2} \lambda^{\prime}+\frac{1}{64} n_{k}^{4}\left(\lambda^{\prime}\right)^{2}-\frac{3}{512} n_{k}^{6}\left(\lambda^{\prime}\right)^{3}\right] \\
& +\frac{1}{J} \sum_{\substack{j, k=1 \\
j \neq k}}^{M} N_{j} N_{k}\left[-\frac{1}{32} n_{j}^{2} n_{k}^{2}\left(\lambda^{\prime}\right)^{2}+\frac{5}{1024} n_{j}^{2} n_{k}^{2}\left(n_{j}^{2}+n_{k}^{2}\right)\left(\lambda^{\prime}\right)^{3}\right] \\
& +O\left(\left(\lambda^{\prime}\right)^{4}\right) .
\end{aligned}
$$

\footnotetext{
${ }^{5}$ We thank Matthias Staudacher for clarification on this point.
} 
Comparing with the corresponding expansion in the near-pp-wave limit of the open-string $\mathfrak{s l}(2)$ Neumann sector in eqn. (2.33), we again find precise agreement at one- and two-loop order in $\lambda^{\prime}$, followed by a mismatch at three loops and beyond.

\section{Summary and discussion}

We have formulated a set of long-range Bethe ansätze for open string states that arise from standard deformations of IIB string theory on $A d S_{5} \times S^{5}{ }^{6}$ In a bosonic symmetric-traceless $S^{5}$ sector we studied open string states with Dirichlet boundary conditions, labeled by an $\mathfrak{s u}(2)$ subalgebra of $\mathfrak{p} \mathfrak{s u}(2,2 \mid 4)$. The open-string Bethe ansatz is formulated in this sector from the closed-string scattering matrix $S_{S^{5}}^{\mathrm{IIB}}\left(p_{k}, p_{j}\right)$ in eqn. (3.13) according to

$$
\begin{aligned}
\text { closed } \mathfrak{s u}(2) \text { strings : } & e^{i p_{k} L}=\prod_{j \neq k} S_{S^{5}}^{\mathrm{IIB}}\left(p_{k}, p_{j}\right) \\
\text { open } \mathfrak{s u}(2) \text { strings : } & e^{2 i p_{k} L}=\prod_{j \neq k} S_{S^{5}}^{\mathrm{IIB}}\left(p_{k}, p_{j}\right) S_{S^{5}}^{\mathrm{IIB}}\left(p_{k},-p_{j}\right) .
\end{aligned}
$$

In this sector we found that there is no phase contribution from boundary scattering. The corresponding prescription in the sector of $\mathfrak{s l}(2)$ symmetric-traceless open string states fluctuating in $A d S_{5}$ with Neumann boundary conditions was found to be

$$
\begin{aligned}
\text { closed } \mathfrak{s l}(2) \text { strings : } & e^{i p_{k} L}=\prod_{j \neq k} S_{A d S}^{\mathrm{IIB}}\left(p_{k}, p_{j}\right) \\
\text { open } \mathfrak{s l}(2) \text { strings : } & e^{2 i p_{k} L}=e^{-2 i p_{k}} \prod_{j \neq k} S_{A d S}^{\mathrm{IIB}}\left(p_{k}, p_{j}\right) S_{A d S}^{\mathrm{IIB}}\left(p_{k},-p_{j}\right) .
\end{aligned}
$$

The closed-string scattering matrix $S_{A d S}^{\mathrm{IIB}}\left(p_{k}, p_{j}\right)$ appears in eqn. (3.33) above. In this sector the open-string Bethe equation receives a contribution that may be interpreted as being due to boundary scattering; this appears as the $e^{-2 i p_{k}}$ prefactor on the right-hand side of eqn. (5.4). In both sectors we computed the near-pp-wave energy spectra of general $N$ impurity states directly from the string theory. The Bethe ansätze presented here reproduce these results precisely, which is consistent with the expectations of integrability.

When the above prescription is applied to the corresponding sectors of operators in the dual $\mathcal{N}=2$ defect conformal field theory, we obtain

$$
\begin{aligned}
\text { closed } \mathfrak{s u}(2) \text { SYM spin chains : } & e^{i p_{k} L}=\prod_{j \neq k} S_{\mathfrak{s u}(2)}\left(p_{k}, p_{j}\right), \\
\text { open } \mathfrak{s u}(2) \text { SYM spin chains : } & e^{2 i p_{k} L}=\prod_{j \neq k} S_{\mathfrak{s u}(2)}\left(p_{k}, p_{j}\right) S_{\mathfrak{s u}(2)}\left(p_{k},-p_{j}\right),
\end{aligned}
$$

\footnotetext{
${ }^{6}$ Let us note that our methods may also be useful in more general deformations of the theory, some examples of which were considered recently in [6].
} 
in the $\mathfrak{s u}(2)$ sector, and

$$
\begin{aligned}
\text { closed } \mathfrak{s l}(2) \text { SYM spin chains : } & e^{i p_{k} L}=\prod_{j \neq k} S_{\mathfrak{s l}(2)}\left(p_{k}, p_{j}\right), \\
\text { open } \mathfrak{s l}(2) \text { SYM spin chains : } & e^{2 i p_{k} L}=e^{-2 i p_{k}} \prod_{j \neq k} S_{\mathfrak{s l}(2)}\left(p_{k}, p_{j}\right) S_{\mathfrak{s l}(2)}\left(p_{k},-p_{j}\right),
\end{aligned}
$$

in the $\mathfrak{s l}(2)$ sector. We find that the operator anomalous dimensions (or energy spectra of the corresponding spin chains) in both the $\mathcal{N}=4$ (5.5, 5.7) and $\mathcal{N}=2$ (5.6, 5.8) gauge theories match the energy spectra of general $\mathfrak{s u}(2)$ and $\mathfrak{s l}(2)$ physical string states to oneand two-loop order in $\lambda^{\prime}$, but disagree at three-loop order and higher.

In 31, Beisert, Dippel and Staudacher suggested a generic type of term that could lead to order-of-limits issues in the comparison of string and gauge theory in this setting: ${ }^{7}$

$$
\frac{\lambda^{L}}{(c+\lambda)^{L}}
$$

Terms of this form will lead to different contributions depending on the sequence of limits taken in figure 1 above. While the range of interactions associated with such terms would typically be greater than the length of the chain, they would not necessarily have to wrap around the spin chain: they need only involve a number of vertices that exceeds the total number of lattice sites on the chain. For closed chains, this could obviously include wrapping interactions, pictured schematically in figure 3. For both open and closed chains, however,

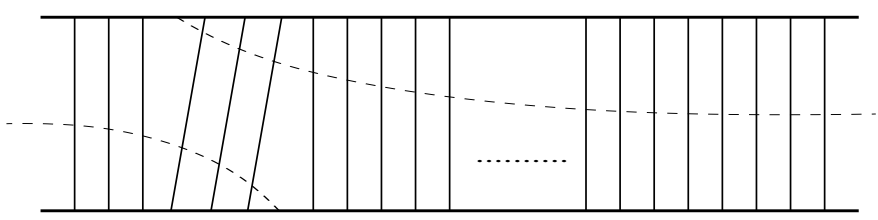

Figure 3: Wrapping interaction diagram

these interactions might also be of the form shown in figure 4, which does not require

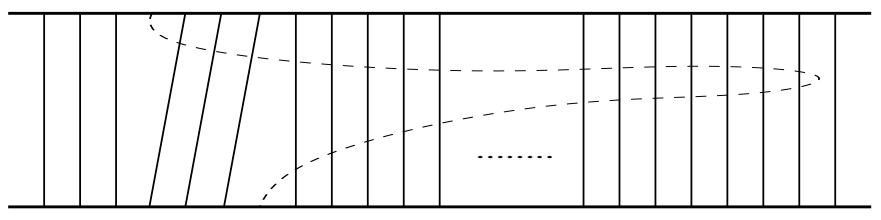

Figure 4: A long-range, non-wrapping diagram

periodicity on the lattice. We note that, by definition, both types of diagram are absent

\footnotetext{
${ }^{7}$ Apart from the fact that such terms might explain the mismatch with string theory beyond two loops, there is no other reason to suspect that the gauge theory produces such functions. See 31] for details.
} 
in the limit of infinite chain length. (Other examples include interactions that are defined to stretch between the boundaries of an open chain, or contain a large number of vertices associated with other intermediate states.)

In the $\mathfrak{s u}(2)$ sector, the difference between the closed-chain scattering matrices for the string and gauge theory is collected into the phase $\Phi\left(p_{k}, p_{j}\right)$ :

$$
S_{S^{5}}^{\mathrm{IIB}}\left(p_{k}, p_{j}\right)=S_{\mathfrak{s u}(2)}\left(p_{k}, p_{j}\right) \Phi\left(p_{k}, p_{j}\right)
$$

If the general disagreement between string and gauge theory is due to wrapping interactions alone, we should expect that the contribution from this phase correction to the open-string Bethe ansatz

$$
\Phi\left(p_{k}, p_{j}\right) \Phi\left(p_{k},-p_{j}\right)
$$

will drop out. While some terms from $\Phi\left(p_{k}, p_{j}\right)$ do cancel in eqn. (15.6), there is still a nonzero contribution from these phases to the open string energy spectrum (see eqns. (3.18) and $(3.24)$ ). One conjecture is that this observation in fact isolates stringy contributions to the Bethe equations from each type of interaction pictured in figures 3 and 4 . Contributions from long-range wrapping (figure 3) and non-wrapping (figure 4) interactions might then be encoded by those terms in $\Phi\left(p_{k}, p_{j}\right)$ that do or do not cancel in the phase correction (5.10) to the open-string Bethe equations, respectively.

Another interpretation of our results is that the prescription for extending the closedstring, long-range Bethe ansatz to open strings (5.2, 5.4) cannot be carried over analogously to the dual gauge theory (5.6, 5.8). It is a logical possibility that the discrepancy between the closed-string and closed-chain energy spectra is due solely to wrapping interactions, and that in moving to the open-chain formulation of the gauge theory we have eliminated all orderof-limits issues, but at the same time introduced some other set of unwanted contributions. While this seems unlikely, a direct higher-loop gauge theory calculation might settle any uncertainty.

\section{Acknowledgments}

We would like to thank John Schwarz and Curt Callan for their ongoing support and encouragement. We also thank Niklas Beisert, Andrei Mikhailov and Matthias Staudacher for explanations and useful discussions. This work was supported by US Department of Energy grant DE-FG03-92-ER40701.

\section{References}

[1] D. Berenstein, J. M. Maldacena, and H. Nastase, "Strings in flat space and pp waves from $\mathcal{N}=4$ super Yang Mills," JHEP 04 (2002) 013, hep-th/0202021. 
[2] E. Witten, "Anti-de Sitter space and holography," Adv. Theor. Math. Phys. 2 (1998) 253-291, hep-th/9802150.

[3] S. S. Gubser, I. R. Klebanov, and A. M. Polyakov, "Gauge theory correlators from non-critical string theory," Phys. Lett. B428 (1998) 105-114, hep-th/9802109.

[4] J. M. Maldacena, "The large $N$ limit of superconformal field theories and supergravity," Adv. Theor. Math. Phys. 2 (1998) 231-252, hep-th/9711200.

[5] D. Berenstein, E. Gava, J. M. Maldacena, K. S. Narain, and H. Nastase, "Open strings on plane waves and their Yang-Mills duals," hep-th/0203249.

[6] B. Chen, X. J. Wang, and Y. S. Wu, "Integrable open spin chain in super Yang-Mills and the plane-wave / SYM duality," JHEP 02 (2004) 029, hep-th/0401016.

[7] O. DeWolfe and N. Mann, "Integrable open spin chains in defect conformal field theory," JHEP 04 (2004) 035, hep-th/0401041.

[8] P. Lee and J.-w. Park, "Open strings in PP-wave background from defect conformal field theory," Phys. Rev. D67 (2003) 026002, hep-th/0203257.

[9] J. Stefanski, B., "Open spinning strings," JHEP 03 (2004) 057, hep-th/0312091.

[10] Y. Susaki, Y. Takayama, and K. Yoshida, "Open semiclassical strings and long defect operators in AdS/dCFT correspondence," hep-th/0410139.

[11] B. Chen, X.-J. Wang, and Y.-S. Wu, "Open spin chain and open spinning string," Phys. Lett. B591 (2004) 170-180, hep-th/0403004.

[12] V. Balasubramanian, M.-x. Huang, T. S. Levi, and A. Naqvi, "Open strings from $\mathcal{N}=4$ super Yang-Mills," JHEP 08 (2002) 037, hep-th/0204196.

[13] H. Takayanagi and T. Takayanagi, "Open strings in exactly solvable model of curved space-time and pp-wave limit," JHEP 05 (2002) 012, hep-th/0204234.

[14] Y. Imamura, "Open string: BMN operator correspondence in the weak coupling regime," Prog. Theor. Phys. 108 (2003) 1077-1097, hep-th/0208079.

[15] K. Skenderis and M. Taylor, "Open strings in the plane wave background. I: Quantization and symmetries," Nucl. Phys. B665 (2003) 3-48, hep-th/0211011.

[16] K. Skenderis and M. Taylor, "Open strings in the plane wave background. II: Superalgebras and spectra," JHEP 07 (2003) 006, hep-th/0212184.

[17] A. Parnachev and A. V. Ryzhov, "Strings in the near plane wave background and AdS/CFT," JHEP 10 (2002) 066, hep-th/0208010. 
[18] C. G. Callan, Jr., H. K. Lee, T. McLoughlin, J. H. Schwarz, I. Swanson, and X. Wu, "Quantizing string theory in $A d S_{5} \times S^{5}$ : Beyond the pp-wave," Nucl. Phys. B673 (2003) 3-40, hep-th/0307032.

[19] C. G. Callan, Jr., T. McLoughlin, and I. Swanson, "Holography beyond the Penrose limit," Nucl. Phys. B694 (2004) 115-169, hep-th/0404007.

[20] C. G. Callan, Jr., T. McLoughlin, and I. Swanson, "Higher impurity AdS/CFT correspondence in the near-BMN limit," Nucl. Phys. B700 (2004) 271-312, hep-th/0405153.

[21] I. Swanson, "On the integrability of string theory in $A d S_{5} \times S^{5}$," hep-th/0405172.

[22] T. McLoughlin and I. Swanson, "N-impurity superstring spectra near the pp-wave limit," Nucl. Phys. B702 (2004) 86-108, hep-th/0407240.

[23] I. Swanson, "Quantum string integrability and AdS/CFT," Nucl. Phys. B709 (2005) 443-464, hep-th/0410282.

[24] M. Blau, J. Figueroa-O'Farrill, C. Hull, and G. Papadopoulos, "A new maximally supersymmetric background of IIB superstring theory," JHEP 01 (2002) 047, hep-th/0110242.

[25] M. Blau, J. Figueroa-O'Farrill, C. Hull, and G. Papadopoulos, "Penrose limits and maximal supersymmetry," Class. Quant. Grav. 19 (2002) L87-L95, hep-th/0201081.

[26] M. Blau, J. Figueroa-O'Farrill, and G. Papadopoulos, "Penrose limits, supergravity and brane dynamics," Class. Quant. Grav. 19 (2002) 4753, hep-th/0202111.

[27] N. Beisert, S. Frolov, M. Staudacher, and A. A. Tseytlin, "Precision spectroscopy of AdS/CFT," JHEP 10 (2003) 037, hep-th/0308117.

[28] A. A. Tseytlin, "Spinning strings and AdS/CFT duality," hep-th/0311139.

[29] G. Arutyunov and M. Staudacher, "Two-loop commuting charges and the string / gauge duality," hep-th/0403077.

[30] J. A. Minahan, "Higher loops beyond the SU(2) sector," JHEP 10 (2004) 053, hep-th/0405243.

[31] N. Beisert, V. Dippel, and M. Staudacher, "A novel long range spin chain and planar $\mathcal{N}=4$ super Yang- Mills," JHEP 07 (2004) 075, hep-th/0405001.

[32] I. R. Klebanov, M. Spradlin, and A. Volovich, "New effects in gauge theory from pp-wave superstrings," Phys. Lett. B548 (2002) 111-118, hep-th/0206221.

[33] D. Serban and M. Staudacher, "Planar $\mathcal{N}=4$ gauge theory and the Inozemtsev long range spin chain," JHEP 06 (2004) 001, hep-th/0401057. 
[34] J. A. Minahan and K. Zarembo, "The Bethe-ansatz for $\mathcal{N}=4$ super Yang-Mills," JHEP 03 (2003) 013, hep-th/0212208.

[35] N. Beisert, C. Kristjansen, and M. Staudacher, "The dilatation operator of $\mathcal{N}=4$ super Yang-Mills theory," Nucl. Phys. B664 (2003) 131-184, hep-th/0303060.

[36] N. Beisert, "The $\mathfrak{s u ( 2 | 3 ) ~ d y n a m i c ~ s p i n ~ c h a i n , " ~ N u c l . ~ P h y s . ~ B 6 8 2 ~ ( 2 0 0 4 ) ~ 4 8 7 - 5 2 0 , ~}$ hep-th/0310252.

[37] N. Beisert, "The dilatation operator of $\mathcal{N}=4$ super Yang-Mills theory and integrability," Phys. Rept. 405 (2005) 1-202, hep-th/0407277.

[38] N. Beisert, "Higher-loop integrability in $\mathcal{N}=4$ gauge theory," Comptes Rendus Physique 5 (2004) 1039-1048, hep-th/0409147.

[39] O. DeWolfe, D. Z. Freedman, and H. Ooguri, "Holography and defect conformal field theories," Phys. Rev. D66 (2002) 025009, hep-th/0111135.

[40] S. G. Naculich, H. J. Schnitzer, and N. Wyllard, "pp-wave limits and orientifolds," Nucl. Phys. B650 (2003) 43-74, hep-th/0206094.

[41] R. R. Metsaev, "Type IIB Green-Schwarz superstring in plane wave Ramond- Ramond background," Nucl. Phys. B625 (2002) 70-96, hep-th/0112044

[42] R. R. Metsaev and A. A. Tseytlin, "Exactly solvable model of superstring in plane wave Ramond- Ramond background," Phys. Rev. D65 (2002) 126004, hep-th/0202109.

[43] A. Dabholkar and S. Parvizi, "Dp branes in pp-wave background," Nucl. Phys. B641 (2002) 223-234, hep-th/0203231.

[44] G. Mandal, N. V. Suryanarayana, and S. R. Wadia, "Aspects of semiclassical strings in $A d S_{5}, "$ Phys. Lett. B543 (2002) 81-88, hep-th/0206103.

[45] I. Bena, J. Polchinski, and R. Roiban, "Hidden symmetries of the $A d S_{5} \times S^{5}$ superstring," Phys. Rev. D69 (2004) 046002, hep-th/0305116.

[46] G. Arutyunov and M. Staudacher, "Matching higher conserved charges for strings and spins," JHEP 03 (2004) 004, hep-th/0310182.

[47] V. A. Kazakov, A. Marshakov, J. A. Minahan, and K. Zarembo, "Classical / quantum integrability in AdS/CFT," JHEP 05 (2004) 024, hep-th/0402207.

[48] V. A. Kazakov and K. Zarembo, "Classical / quantum integrability in non-compact sector of AdS/CFT," JHEP 10 (2004) 060, hep-th/0410105.

[49] N. Beisert, V. A. Kazakov, and K. Sakai, "Algebraic curve for the $S O(6)$ sector of AdS/CFT," hep-th/0410253. 
[50] S. Schafer-Nameki, "The algebraic curve of 1-loop planar $\mathcal{N}=4 \mathrm{SYM}$," hep-th/0412254.

[51] N. Beisert, V. A. Kazakov, K. Sakai, and K. Zarembo, "The Algebraic Curve of Classical Superstrings on $A d S_{5} \times S^{5}$, , hep-th/0502226.

[52] N. Berkovits, "Quantum consistency of the superstring in $A d S_{5} \times S^{5}$ background," hep-th/0411170.

[53] G. Arutyunov and S. Frolov, "Integrable Hamiltonian for classical strings on $A d S_{5} \times S^{5}, " J H E P 02$ (2005) 059, hep-th/0411089.

[54] L. F. Alday, G. Arutyunov, and A. A. Tseytlin, "On integrability of classical superstrings in $A d S_{5} \times S^{5}, "$ hep-th/0502240.

[55] G. Arutyunov and M. Zamaklar, "Linking Baecklund and monodromy charges for strings on $A d S_{5} \times S^{5}, "$ hep-th/0504144.

[56] K. Peeters, J. Plefka, and M. Zamaklar, "Splitting spinning strings in AdS/CFT," JHEP 11 (2004) 054, hep-th/0410275.

[57] K. Peeters, J. Plefka, and M. Zamaklar, "Splitting strings and chains," Fortsch. Phys. 53 (2005) 640-646, hep-th/0501165.

[58] J. A. Minahan, "The $S U(2)$ sector in AdS/CFT," hep-th/0503143.

[59] M. Staudacher, "The factorized S-matrix of CFT/AdS," hep-th/0412188.

[60] C. G. Callan, Jr., J. Heckman, T. McLoughlin, and I. Swanson, "Lattice super Yang-Mills: A virial approach to operator dimensions," Nucl. Phys. B701 (2004) 180-206, hep-th/0407096.

[61] N. Beisert and M. Staudacher, "The $\mathcal{N}=4$ SYM integrable super spin chain," Nucl. Phys. B670 (2003) 439-463, hep-th/0307042.

[62] V. I. Inozemtsev, "On the connection between the one-dimensional $s=1 / 2$ Heisenberg chain and Haldane Shastry model," J. Stat. Phys. 59 (1990) 1143. JINR-E5-89-490.

[63] V. I. Inozemtsev, "Integrable Heisenberg-van Vleck chains with variable range exchange," Phys. Part. Nucl. 34 (2003) 166-193, hep-th/0201001.

[64] G. Arutyunov, S. Frolov, and M. Staudacher, "Bethe ansatz for quantum strings," JHEP 10 (2004) 016, hep-th/0406256.

[65] B. S. Shastry and A. Dhar, "Solution of a generalized Stieltjes problem," cond-mat/010144.

[66] S. A. Frolov, R. Roiban, and A. A. Tseytlin, "Gauge - string duality for superconformal deformations of $\mathcal{N}=4$ super Yang-Mills theory," hep-th/0503192. 